\title{
On the Relationship between Classical and Deformed Hopf Fibrations
}

\author{
Tomasz BRZEZIŃSKI †๋, James GAUNT ${ }^{\S}$ and Alexander SCHENKEL $\S$ \\ $\dagger$ Department of Mathematics, Swansea University, Swansea University Bay Campus, \\ Fabian Way, Swansea SA1 8EN, UK \\ E-mail: T.Brzezinski@swansea.ac.uk \\ $\ddagger$ Faculty of Mathematics, University of Biatystok, \\ K. Ciotkowskiego 1M, 15-245 Biatystok, Poland \\ $\S$ School of Mathematical Sciences, University of Nottingham, \\ University Park, Nottingham NG7 2RD, UK \\ E-mail: james.gaunt@nottingham.ac.uk,alexander.schenkel@nottingham.ac.uk
}

Received September 17, 2019, in final form February 17, 2020; Published online February 23, 2020 https://doi.org/10.3842/SIGMA.2020.008

\begin{abstract}
The $\theta$-deformed Hopf fibration $\mathbb{S}_{\theta}^{3} \rightarrow \mathbb{S}^{2}$ over the commutative 2-sphere is compared with its classical counterpart. It is shown that there exists a natural isomorphism between the corresponding associated module functors and that the affine spaces of classical and deformed connections are isomorphic. The latter isomorphism is equivariant under an appropriate notion of infinitesimal gauge transformations in these contexts. Gauge transformations and connections on associated modules are studied and are shown to be sensitive to the deformation parameter. A homotopy theoretic explanation for the existence of a close relationship between the classical and deformed Hopf fibrations is proposed.
\end{abstract}

Key words: noncommutative geometry; principal comodule algebras; noncommutative principal bundles; Hopf fibrations; homotopy equivalence

2020 Mathematics Subject Classification: 81T75; 16T05

To Gianni on the occasion of his 60th birthday

\section{Introduction and summary}

The Hopf fibration $\mathbb{S}^{3} \rightarrow \mathbb{S}^{2}$ is the prime example of a non-trivial principal $U(1)$-bundle over the 2-sphere. From an algebraic perspective, it can be described as a faithfully-flat Hopf-Galois extension, or equivalently as a principal comodule algebra, consisting of the algebra $A=\mathcal{O}\left(\mathbb{S}^{3}\right)$ of functions on $\mathbb{S}^{3}$ together with the canonically induced coaction $\delta: A \rightarrow A \otimes H$ of the Hopf algebra $H=\mathcal{O}(U(1))$ of functions on the structure group $U(1)$. Due to its origin in ordinary geometry, this Hopf-Galois extension is special in the sense that the total space algebra $A$, the structure Hopf algebra $H$ and consequently the base space algebra $B:=A^{\operatorname{co} H} \cong \mathcal{O}\left(\mathbb{S}^{2}\right)$ are commutative.

As Hopf-Galois theory does not require commutative algebras, it provides a natural framework in which to study noncommutative generalizations of principal bundles. In particular, there exists a 1-parameter family of deformations of the Hopf fibration $\mathbb{S}^{3} \rightarrow \mathbb{S}^{2}$, where the total space algebra is deformed to the Connes-Landi 3-sphere $A_{\theta}=\mathcal{O}\left(\mathbb{S}_{\theta}^{3}\right)$ and the structure Hopf algebra $H$ and base space algebra $B$ remain undeformed. It is important to emphasize that even

This paper is a contribution to the Special Issue on Noncommutative Manifolds and their Symmetries in honour of Giovanni Landi. The full collection is available at https://www.emis.de/journals/SIGMA/Landi.html 
though the base space algebra $B$ and structure Hopf algebra $H$ are commutative, these examples are not commutative principal bundles since the total space $A_{\theta}$ is a noncommutative algebra. Hence, one should expect certain, potentially subtle, noncommutative geometry features in these examples.

In this work, we shall study in detail the geometric structures on the deformed Hopf fibrations $\mathbb{S}_{\theta}^{3} \rightarrow \mathbb{S}^{2}$ that are relevant for gauge theory and compare those with the corresponding structures on the classical Hopf fibration $\mathbb{S}^{3} \rightarrow \mathbb{S}^{2}$. An interesting observation is that many, however not all, of these geometric structures coincide for these examples even though $\mathbb{S}_{\theta}^{3} \rightarrow \mathbb{S}^{2}$ and $\mathbb{S}^{3} \rightarrow \mathbb{S}^{2}$ are not isomorphic as Hopf-Galois extensions. This follows as $A_{\theta}=\mathcal{O}\left(\mathbb{S}_{\theta}^{3}\right)$ is a noncommutative algebra whilst $A=\mathcal{O}\left(\mathbb{S}^{3}\right)$ is commutative. In more detail, we prove the following results:

1. The associated module functors for the classical and deformed Hopf fibrations are naturally isomorphic, i.e., the theory of associated modules is insensitive to the deformation parameter $\theta$. In physics terminology, this means that we have the same matter fields on the classical and deformed Hopf fibrations.

2. The affine spaces of connections, with respect to suitable Kähler-type differential calculi, for the classical and deformed Hopf fibrations are isomorphic. It is also shown that this isomorphism is compatible with the action of infinitesimal gauge transformations. In physics terminology, this means that we have the same gauge fields on the classical and deformed Hopf fibrations.

3. In contrast to the previous two points, the action of infinitesimal gauge transformations and connections on associated modules does depend on the deformation parameter. Hence, it is different for the classical and deformed Hopf fibrations. In physics terminology, this means that the coupling of gauge fields to matter fields is sensitive to the deformation parameter.

Even though our direct calculations were able to unravel these striking similarities between the classical and deformed Hopf fibrations, they provide no conceptual reason for why these two non-isomorphic Hopf-Galois extensions should behave similarly in certain respects. As a first step towards a more conceptual explanation, we investigate our examples of Hopf-Galois extensions from the homotopy theoretic perspective proposed by Kassel and Schneider in [29]. We shall show that the classical and deformed Hopf fibrations are homotopy equivalent, however in a slightly different way as the one proposed by Kassel and Schneider. In more detail, while the interval object in [29] is modelled by the polynomial algebra $\mathbb{C}[y]$ of the affine line, we require an interval object that is modelled by a larger algebra that also contains exponential functions. This is related to the fact that the deformation parameter $\theta$, which we would like to turn to zero by a homotopy equivalence, enters the deformed Hopf fibration in an exponential form $q=\mathrm{e}^{2 \pi \mathrm{i} \theta}$. We give some indications, however not a full proof, that this homotopy equivalence could be the reason why the classical and deformed Hopf fibrations have naturally isomorphic associated module functors. A detailed study of these aspects, and in particular of the interplay between homotopy equivalence and connections, is beyond the scope of this paper. However, we hope to come back to this issue in a future work.

The outline of the remainder of this paper is as follows: In Section 2 we provide a brief review of the theory of Hopf-Galois extensions and principal comodule algebras. We also introduce the examples of interest in this work, namely the classical Hopf fibration $\mathbb{S}^{3} \rightarrow \mathbb{S}^{2}$ and its deformation $\mathbb{S}_{\theta}^{3} \rightarrow \mathbb{S}^{2}$ by a suitable family of 2 -cocycles. In Section 3 we describe the associated module functors for both the classical and deformed Hopf fibrations and prove that they are naturally isomorphic functors. We would like to emphasize that this natural isomorphism is a specific feature of our particular example and not a consequence of the general theory of 2cocycle deformations, see Remark 3.4. Section 4 starts with a brief review of the theory of Atiyah 
sequences and connections on principal comodule algebras. We shall discuss both the case of universal differential calculi and also the more general case of concordant differential calculi on principal comodule algebras. We then describe in detail the Atiyah sequence for universal and Kähler-type differential calculi on the classical and deformed Hopf fibrations and construct an isomorphism between the affine spaces of classical and deformed connections. Again, we emphasize that this isomorphism results from the specific example under discussion and not from the theory of 2-cocycle deformations, see Remark 4.13. It is also shown that this isomorphism is compatible with the action of infinitesimal gauge transformations. In Section 5 we study gauge transformations and connections on the associated modules of the classical and deformed Hopf fibrations and show that these structures are sensitive to the deformation parameter. In Section 6 we show that the classical and deformed Hopf fibrations are in a suitable sense homotopy equivalent and give some indications why this should imply the properties of associated modules described in Section 3.

Notation and conventions: Throughout the bulk of the paper, by an algebra we mean an associative and unital algebra over a field $k$. Unless otherwise stated, $k=\mathbb{C}$, the field of complex numbers. The exception is Section 6 where algebras over commutative rings are also admitted. The multiplication map in an algebra $A$ is denoted by $\mu_{A}: A \otimes A \rightarrow A$ and the unit map by $\eta_{A}: k \rightarrow A$. The unit element $\mathbf{1}_{A} \in A$, or simply $\mathbf{1} \in A$, is obtained by evaluating the unit map on $1 \in k$. We denote the category of left $A$-modules by ${ }_{A} \mathscr{M}$ and that of right $A$-modules by $\mathscr{M}_{A}$. The full subcategory of finitely generated projective left $A$-modules is denoted by ${ }_{A} \mathscr{P} \subseteq{ }_{A} \mathscr{M}$.

The comultiplication in a Hopf algebra $H$ is denoted by $\Delta$ (or $\Delta_{H}$ if not sufficiently clear from the context), the counit by $\epsilon$ (or $\epsilon_{H}$ ), and the antipode by $S$ (or $S_{H}$ ). We always assume that $S$ is a bijective map. For comultiplications $\Delta: H \rightarrow H \otimes H$, right $H$-coactions $\delta: V \rightarrow V \otimes H$ and left $H$-coactions $\rho: V \rightarrow H \otimes V$, we use the following variant of Sweedler's notation (with suppressed summation)

$$
\Delta(h)=h_{\underline{1}} \otimes h_{\underline{2}}, \quad \delta(v)=v_{\underline{0}} \otimes v_{\underline{1}}, \quad \rho(v)=v_{\underline{-1}} \otimes v_{\underline{0}} .
$$

The category of left $H$-comodules is denoted by ${ }^{H} \mathscr{M}$ and that of right $H$-comodules by $\mathscr{M}^{H}$. The category of finite-dimensional left $H$-comodules is denoted by ${ }^{H} \mathscr{M}_{\text {fin }}$.

\section{Noncommutative Hopf fibrations}

\section{$2.1 \quad$ Principal comodule algebras}

The study of connections on noncommutative principal bundles has been initiated in [16] and developed further by the introduction of strong connections in [24, 26]. This framework has been extended beyond Hopf algebras in $[17,18]$ and then formalized in terms of principal coalgebra extensions in [14] and principal comodule algebras in [28].

Definition 2.1. Let $H$ be a Hopf algebra with bijective antipode. A right $H$-comodule algebra $(A, \delta)$ is called a principal comodule algebra if it admits a strong connection, i.e., a linear map $\ell: H \rightarrow A \otimes A$, such that

$$
\begin{array}{ll}
\ell\left(\mathbf{1}_{H}\right)=\mathbf{1}_{A} \otimes \mathbf{1}_{A} & \text { (normalization), } \\
\mu_{A} \circ \ell=\eta_{A} \circ \epsilon_{H} & \text { (splitting property), } \\
(\mathrm{id} \otimes \delta) \circ \ell=(\ell \otimes \mathrm{id}) \circ \Delta_{H} & \text { (right colinearity), } \\
\left(\delta_{S} \otimes \mathrm{id}\right) \circ \ell=(\mathrm{id} \otimes \ell) \circ \Delta_{H} & \text { (left colinearity), }
\end{array}
$$

where $\delta: A \rightarrow A \otimes H$ is the right $H$-coaction and $\delta_{S}: A \rightarrow H \otimes A$ is the associated left $H$-coaction defined by

$$
\delta_{S}:=\left(S^{-1} \otimes \mathrm{id}\right) \circ \text { flip } \circ \delta: a \longmapsto S^{-1}\left(a_{\underline{1}}\right) \otimes a_{\underline{0}} .
$$


In the context of noncommutative geometry, principal comodule algebras are interpreted as principal bundles. The algebra $A$ is the algebra of functions on the (noncommutative) total space, the Hopf algebra $H$ is the structure (quantum) group and the subalgebra of coinvariants

$$
B:=A^{\mathrm{co} H}:=\left\{a \in A: \delta(a)=a \otimes \mathbf{1}_{H}\right\} \subseteq A
$$

is the algebra of functions on the (noncommutative) base space. Note that since $\delta$ is an algebra homomorphism, $B$ is indeed a subalgebra of $A$. The existence of a strong connection ensures that $A$ is a Hopf-Galois extension of $B$, i.e., that the canonical Galois map

$$
\text { can }:=\left(\mu_{A} \otimes \mathrm{id}\right) \circ(\mathrm{id} \otimes \delta): A \otimes_{B} A \longrightarrow A \otimes H, \quad a \otimes_{B} a^{\prime} \longmapsto a a_{\underline{0}}^{\prime} \otimes a_{\underline{1}}^{\prime}
$$

is bijective. Explicitly, the inverse of the canonical Galois map is given by the composite of

$$
A \otimes H \stackrel{\mathrm{id} \otimes \ell}{\longrightarrow} A \otimes A \otimes A \stackrel{\mu_{A} \otimes \mathrm{id}}{\longrightarrow} A \otimes A \longrightarrow A \otimes_{B} A .
$$

The Galois property encodes freeness of the action of the structure (quantum) group.

The existence of a strong connection also implies that $A$ is an $H$-equivariantly projective left $B$-module, i.e., the restriction of the multiplication map to $B \otimes A$ has a right $H$-comodule left $B$-module splitting. Explicitly,

$$
\sigma:=\left(\mu_{A} \otimes \mathrm{id}\right) \circ(\mathrm{id} \otimes \ell) \circ \delta: A \longrightarrow B \otimes A .
$$

In fact, a principal comodule algebra is the same as an $H$-equivariantly projective Hopf-Galois extension. The projectivity property gives the notion of a principal comodule algebra full geometric meaning, as it implies that $A$ admits a noncommutative connection in the sense of [23]. This is in perfect concord with Cartan's definition of a principal action of a compact Lie group [20]. We return to these differential geometric aspects of principal comodule algebras in Section 4.

Furthermore, a principal comodule algebra is the same as a faithfully-flat Hopf-Galois extension, i.e., a Hopf-Galois extension $A$ of $B$ such that the tensor product functor $(-) \otimes_{B} A: \mathscr{M}_{B} \rightarrow$ $\mathscr{M}_{A}$ both preserves and reflects exact sequences. This gives an algebraic geometry flavour to the notion of a principal comodule algebra, as it leads to the faithfully flat descent property. As observed by H.-J. Schneider, in one of the key results of Hopf-Galois theory [33, Theorem I], if $H$ admits an invariant integral (i.e., $H$ is coseparable) such as the Haar measure on the coordinate algebra of a compact quantum group [34], then surjectivity of the canonical Galois map (2.2) implies its injectivity as well as faithful-flatness of $A$ as a left $B$-module. An explicit construction of a strong connection, in the more general situation of extensions by coalgebras, is given in [7]. As a consequence, Hopf-Galois extensions given by typical Hopf algebras that feature in noncommutative geometry, and in particular those found in the present text, are automatically principal comodule algebras.

The reader interested in studying further the meaning of principal comodule algebras in classical geometry is encouraged to consult [6].

\subsection{The classical Hopf fibration}

The Hopf fibration $\mathbb{S}^{3} \rightarrow \mathbb{S}^{2}$ can be described algebraically as follows. The total space is given by the $*$-algebra $A=\mathcal{O}\left(\mathbb{S}^{3}\right)$ of functions on the algebraic 3 -sphere. Concretely, $A$ is the commutative $*$-algebra generated by $z_{1}$ and $z_{2}$, modulo the $*$-ideal generated by the 3 -sphere relation

$$
z_{1}^{*} z_{1}+z_{2}^{*} z_{2}=\mathbf{1}
$$


The structure group is described by the *-Hopf algebra $H=\mathcal{O}(U(1))$ of functions on the algebraic circle group $U(1)$. Concretely, $H$ is the commutative $*$-algebra generated by $t$, modulo the $*$-ideal generated by the circle relation

$$
t^{*} t=1
$$

The coproduct, counit and antipode read as

$$
\Delta(t)=t \otimes t, \quad \epsilon(t)=1, \quad S(t)=t^{*} .
$$

We endow $A$ with the structure of a right $H$-comodule $*$-algebra by defining the right $H$-coaction $\delta: A \rightarrow A \otimes H$ on the generators as

$$
\delta\left(z_{1}\right)=z_{1} \otimes t, \quad \delta\left(z_{2}\right)=z_{2} \otimes t .
$$

The compatibility condition $\delta \circ *=(* \otimes *) \circ \delta$ between the coaction and $*$-involution gives

$$
\delta\left(z_{1}^{*}\right)=z_{1}^{*} \otimes t^{*}, \quad \delta\left(z_{2}^{*}\right)=z_{2}^{*} \otimes t^{*} .
$$

Lemma 2.2. The *-subalgebra of $H$-coinvariants

$$
B:=A^{\mathrm{co} H}:=\{a \in A: \delta(a)=a \otimes \mathbf{1}\} \subseteq A
$$

is isomorphic to the $*$-algebra $\mathcal{O}\left(\mathbb{S}^{2}\right)$ of functions on the algebraic 2-sphere.

Proof. We find that $A^{\mathrm{co} H} \subseteq A$ is generated as a *-algebra by

$$
z:=2 z_{1} z_{2}^{*} \in A^{\mathrm{coH}}, \quad x:=z_{1}^{*} z_{1}-z_{2}^{*} z_{2} \in A^{\mathrm{co} H} .
$$

These generators satisfy the relations

$$
x^{*}=x, \quad z^{*} z+x^{2}=\mathbf{1},
$$

hence $A^{\mathrm{co} H} \cong \mathcal{O}\left(\mathbb{S}^{2}\right)$.

Proposition 2.3. The right $H$-comodule $*$-algebra $(A, \delta)$ described above is a principal comodule algebra.

Proof. This is a special case of what was proven in [19]. We note in passing that a strong connection can be defined iteratively by

$$
\ell(\mathbf{1})=\mathbf{1} \otimes \mathbf{1}, \quad \ell\left(t^{n}\right)= \begin{cases}z_{1}^{*} \ell\left(t^{n-1}\right) z_{1}+z_{2}^{*} \ell\left(t^{n-1}\right) z_{2}, & \text { for } n>0, \\ z_{1} \ell\left(t^{n+1}\right) z_{1}^{*}+z_{2} \ell\left(t^{n+1}\right) z_{2}^{*}, & \text { for } n<0,\end{cases}
$$

for all $n \in \mathbb{Z}$.

\section{$2.3 \quad 2$-cocycle deformations}

We construct a 1-parameter family of noncommutative Hopf fibrations via 2-cocyle deformations. We refer the reader to [1] for a general cocycle deformation framework and also to [10, 11, 31] for the more specific case of toric deformations, which is sufficient for our present work. Let $K=\mathcal{O}\left(\mathbb{T}^{2}\right)$ denote the $*$-Hopf algebra of functions on the algebraic 2-torus. As a vector space, $K$ is spanned by the basis

$$
\left\{t_{\mathbf{m}}: \mathbf{m}=\left(m_{1}, m_{2}\right) \in \mathbb{Z}^{2}\right\},
$$


on which the product, unit and involution are defined by

$$
t_{\mathbf{m}} t_{\mathbf{m}^{\prime}}=t_{\mathbf{m}+\mathbf{m}^{\prime}}, \quad \mathbf{1}=t_{\mathbf{0}}, \quad t_{\mathbf{m}}^{*}=t_{-\mathbf{m}} .
$$

The coproduct, counit and antipode are given by

$$
\Delta\left(t_{\mathbf{m}}\right)=t_{\mathbf{m}} \otimes t_{\mathbf{m}}, \quad \epsilon\left(t_{\mathbf{m}}\right)=1, \quad S\left(t_{\mathbf{m}}\right)=t_{\mathbf{m}}^{*}
$$

We endow $A$ with the structure of a left $K$-comodule $*$-algebra by defining the left $K$-coaction $\rho: A \rightarrow K \otimes A$ on the generators as

$$
\rho\left(z_{1}\right)=t_{(1,0)} \otimes z_{1}, \quad \rho\left(z_{2}\right)=t_{(0,1)} \otimes z_{2} .
$$

The compatibility condition $\rho \circ *=(* \otimes *) \circ \rho$ between the coaction and $*$-involution implies

$$
\rho\left(z_{1}^{*}\right)=t_{(-1,0)} \otimes z_{1}^{*}, \quad \rho\left(z_{2}^{*}\right)=t_{(0,-1)} \otimes z_{2}^{*} .
$$

Recalling the right $H$-coaction from (2.3), we observe that $(A, \rho, \delta)$ is a $(K, H)$-bicomodule *-algebra, i.e., the diagram

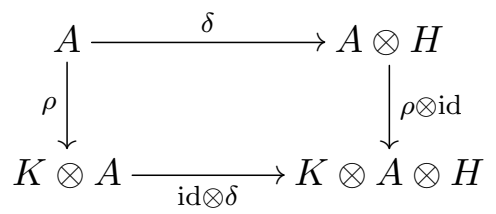

commutes and both $\delta$ and $\rho$ are algebra maps.

Let us recall from, e.g., [32, Section 2.3] that a 2-cocycle on a Hopf algebra $K$ is a convolutioninvertible linear map $\sigma: K \otimes K \rightarrow \mathbb{C}$ that is unital, i.e., $\sigma(a \otimes \mathbf{1})=\epsilon(a)=\sigma(\mathbf{1} \otimes a)$ for all $a \in K$, and that satisfies the cocycle condition

$$
\sigma\left(b_{1} \otimes c_{1}\right) \sigma\left(a \otimes b_{2} c_{2}\right)=\sigma\left(a_{\underline{1}} \otimes b_{1}\right) \sigma\left(a_{\underline{2}} b_{2} \otimes c\right),
$$

for all $a, b, c \in K$. Every 2-cocycle $\sigma$ on $K$ defines a deformation of $K$ into a new Hopf algebra $K_{\sigma}$ as well as a deformation of the $(K, H)$-bicomodule algebra $(A, \rho, \delta)$ into a deformed $\left(K_{\sigma}, H\right)$ bicomodule algebra $\left(A_{\sigma}, \rho, \delta\right)$, see, e.g., [1, Proposition 2.27]. Our focus will be on the family of 2-cocycles defined by

$$
\sigma_{\theta}\left(t_{\mathbf{m}} \otimes t_{\mathbf{m}^{\prime}}\right)=\exp \left(\pi \mathbf{i m}^{\mathrm{T}} \Theta \mathbf{m}^{\prime}\right)=\exp \left(\pi \mathrm{i} \sum_{j, k=1}^{2} m_{j} \Theta^{j k} m_{k}^{\prime}\right),
$$

where

$$
\Theta=\left(\begin{array}{cc}
0 & \theta \\
-\theta & 0
\end{array}\right), \quad \theta \in \mathbb{R}
$$

(We note in passing that (2.8) may also be interpreted as a $U(1)$-valued group 2-cocycle $\widetilde{\sigma}_{\theta}: \mathbb{Z}^{2} \times$ $\mathbb{Z}^{2} \rightarrow U(1),\left(\mathbf{m}, \mathbf{m}^{\prime}\right) \mapsto \sigma_{\theta}\left(t_{\mathbf{m}} \otimes t_{\mathbf{m}^{\prime}}\right)$ on the Pontryagin dual $\left(\mathbb{T}^{2}\right)^{*}=\mathbb{Z}^{2}$.) As $K=\mathcal{O}\left(\mathbb{T}^{2}\right)$ is commutative and cocommutative it follows that $K_{\theta}=K$ as Hopf algebras. The deformed $(K, H)$-bicomodule $*$-algebra $\left(A_{\theta}, \rho, \delta\right)$ is given as follows. As a $(K, H)$-bicomodule, we have that $\left(A_{\theta}, \rho, \delta\right)=(A, \rho, \delta)$, i.e., the left $K$-coaction and right $H$-coaction remain undeformed. The product is deformed to the $\star_{\theta}$-product defined by

$$
a \star_{\theta} a^{\prime}:=\sigma_{\theta}\left(a_{\underline{-1}} \otimes a_{\underline{-1}}^{\prime}\right) a_{\underline{0}} a_{\underline{0}}^{\prime} .
$$


Note that the cocycle condition (2.7) ensures associativity of the $\star_{\theta}$-product. The unit $\mathbf{1}_{\theta}=\mathbf{1}$ and involution $a^{* \theta}=a^{*}$ remain undeformed. Using (2.5), (2.8) and (2.9), one finds the following commutation relations for the generators

$$
\begin{array}{ll}
z_{1} \star_{\theta} z_{1}^{*}=z_{1}^{*} \star_{\theta} z_{1}, & z_{2} \star_{\theta} z_{2}^{*}=z_{2}^{*} \star_{\theta} z_{2}, \\
z_{1} \star_{\theta} z_{2}=q z_{2} \star_{\theta} z_{1}, & z_{1} \star_{\theta} z_{2}^{*}=q^{-1} z_{2}^{*} \star_{\theta} z_{1},
\end{array}
$$

where $q=\mathrm{e}^{2 \pi \mathrm{i} \theta}$. A further calculation shows that

$$
z_{1}^{*} \star_{\theta} z_{1}+z_{2}^{*} \star_{\theta} z_{2}=z_{1}^{*} z_{1}+z_{2}^{*} z_{2}=\mathbf{1},
$$

i.e., $A_{\theta}$ describes the Connes-Landi 3 -sphere $\mathbb{S}_{\theta}^{3}$, see, e.g., [21, 22, 31].

Lemma 2.4. The *-subalgebra $A_{\theta}^{\mathrm{co} H} \subseteq A_{\theta}$ of $H$-coinvariants does not depend on the deformation parameter $\theta$, i.e., $A_{\theta}^{\mathrm{co} H}=A^{\mathrm{co} H}=B \cong \mathcal{O}\left(\mathbb{S}^{2}\right)$ is the commutative 2-sphere.

Proof. Recall from the proof of Lemma 2.2 that the undeformed $*$-algebra $A^{\mathrm{co} H}$ of coinvariants is generated by the elements $z:=2 z_{1} z_{2}^{*}$ and $x:=z_{1}^{*} z_{1}-z_{2}^{*} z_{2}$. Using (2.5), we find that the left $K$-coaction on these elements is

$$
\rho(z)=t_{(1,-1)} \otimes z, \quad \rho\left(z^{*}\right)=t_{(-1,1)} \otimes z^{*}, \quad \rho(x)=\mathbf{1} \otimes x,
$$

which implies via (2.9) and (2.8) that their $\star_{\theta}$-products coincide with their undeformed products.

Proposition 2.5. The right $H$-comodule *-algebra $\left(A_{\theta}, \delta\right)$ described above is a principal comodule algebra.

Proof. This follows directly from Proposition 2.3 and [1, Corollary 3.19]. Explicitly, a strong connection can be defined iteratively by

$$
\ell(\mathbf{1})=\mathbf{1} \otimes \mathbf{1}, \quad \ell\left(t^{n}\right)= \begin{cases}z_{1}^{*} \star_{\theta} \ell\left(t^{n-1}\right) \star_{\theta} z_{1}+z_{2}^{*} \star_{\theta} \ell\left(t^{n-1}\right) \star_{\theta} z_{2}, & \text { for } n>0, \\ z_{1} \star_{\theta} \ell\left(t^{n+1}\right) \star_{\theta} z_{1}^{*}+z_{2} \star_{\theta} \ell\left(t^{n+1}\right) \star_{\theta} z_{2}^{*}, & \text { for } n<0,\end{cases}
$$

for all $n \in \mathbb{Z}$. (Compare this expression with (2.4).) Alternatively, this proposition follows also from [11, Lemma 3.19].

\section{Associated modules}

\subsection{Modules associated to principal comodule algebras}

Let $H$ be a Hopf algebra. Given any right $H$-comodule $(M, \delta: M \rightarrow M \otimes H)$ and left $H$-comodule $(V, \rho: V \rightarrow H \otimes V)$, the cotensor product $M \square_{H} V$ is defined as the equalizer

$$
M \square_{H} V \longrightarrow M \otimes V \underset{\operatorname{id} \otimes \rho}{\stackrel{\delta \otimes i d}{\longrightarrow}} M \otimes H \otimes V
$$

in the category of vector spaces. Explicitly,

$$
M \square_{H} V=\left\{\sum_{j} m_{j} \otimes v_{j} \in M \otimes V: \sum_{j} \delta\left(m_{j}\right) \otimes v_{j}=\sum_{j} m_{j} \otimes \rho\left(v_{j}\right)\right\} .
$$

If $M$ is a $(K, H)$-bicomodule and $V$ is an $(H, L)$-bicomodule for some other Hopf algebras $K$ and $L$, then the cotensor product $M \square_{H} V$ is a $(K, L)$-bicomodule. 
Definition 3.1. Let $(A, \delta)$ be a principal $H$-comodule algebra with coinvariant subalgebra $B=A^{\mathrm{co} H}$ and let $(V, \rho)$ be a left $H$-comodule. Since $\delta$ is a left $B$-module homomorphism, the cotensor product $E_{A}(V):=A \square_{H} V$ is a left $B$-module via

$$
B \otimes E_{A}(V) \longrightarrow E_{A}(V), \quad b \otimes(a \otimes v) \longmapsto(b a) \otimes v .
$$

The corresponding functor

$$
E_{A}:{ }^{H} \mathscr{M} \longrightarrow{ }_{B} \mathscr{M}
$$

is called the associated module functor for the principal comodule algebra $A$.

As explained in [13], if $V$ is finite-dimensional, then $E_{A}(V)$ is a finitely generated projective left $B$-module. In other words, (3.3) restricts to a functor

$$
E_{A}:{ }^{H} \mathscr{M}_{\text {fin }} \longrightarrow{ }_{B} \mathscr{P} .
$$

An idempotent for $E_{A}(V)$ can be explicitly constructed from a strong connection $\ell$ on $A$ and a basis of $V$, see [13] and [9].

\subsection{Modules associated to Hopf fibrations}

The aim of this section is to compare the associated module functor $E_{A_{\theta}}$ for the deformed Hopf fibration $\left(A_{\theta}, \delta\right)$ (cf. Proposition 2.5) with the functor $E_{A}$ for the classical Hopf fibration $(A, \delta)$ (cf. Proposition 2.3). As vector spaces,

$$
E_{A_{\theta}}(V)=A_{\theta} \square_{H} V=A \square_{H} V=E_{A}(V) .
$$

However, the left $B$-actions on these spaces are different. In view of (3.2), the left $B$-module structure of $E_{A}(V)$ comes from the commutative multiplication in $A$, restricted to $B \otimes A$, while the left $B$-module structure of $E_{A_{\theta}}(V)$ uses the noncommutative multiplication by $\star_{\theta}$. We shall prove below that the two functors $E_{A_{\theta}}$ and $E_{A}$ are naturally isomorphic. This means that the theory of associated modules for the deformed Hopf fibration $\left(A_{\theta}, \delta\right)$ is equivalent to that for the classical Hopf fibration. Loosely speaking, it could be said that associated modules do not depend on the deformation parameter $\theta$.

In order to construct this natural isomorphism we have to analyze the two different left $B$ module structures in more detail. For this, it is convenient to decompose the underlying $(K, H)$ bicomodule $\left(A_{\theta}, \rho, \delta\right)=(A, \rho, \delta)$ into irreducible representations. We define the homogeneous $(K, H)$-bicomodules

$$
A^{(\mathbf{m}, n)}:=\left\{a \in A: \rho(a)=t_{\mathbf{m}} \otimes a, \delta(a)=a \otimes t^{n}\right\} \subseteq A,
$$

for all $\mathbf{m}=\left(m_{1}, m_{2}\right) \in \mathbb{Z}^{2}$ and $n \in \mathbb{Z}$.

Lemma 3.2. There exists a decomposition of the $(K, H)$-bicomodule $\left(A_{\theta}, \rho, \delta\right)=(A, \rho, \delta)$ as

$$
A=\bigoplus_{m, n \in \mathbb{Z}} A^{((m+n,-m), n)}
$$

The $\star_{\theta}$-product of homogeneous elements $a \in A^{((m+n,-m), n)}$ and $a^{\prime} \in A^{\left(\left(m^{\prime}+n^{\prime},-m^{\prime}\right), n^{\prime}\right)}$ is

$$
a \star_{\theta} a^{\prime}=\mathrm{e}^{\pi \mathrm{i} \theta\left(m n^{\prime}-n m^{\prime}\right)} a a^{\prime} \in A^{\left(\left(m+m^{\prime}+n+n^{\prime},-m-m^{\prime}\right), n+n^{\prime}\right)} .
$$


Proof. Because $A$ is the $(K, H)$-bicomodule underlying a finitely presented $(K, H)$-bicomodule algebra, there exists a decomposition $A=\bigoplus_{\mathbf{m} \in \mathbb{Z}^{2}, n \in \mathbb{Z}} A^{(\mathbf{m}, n)}$, cf. [4, Lemma A.3]. As the generators are homogeneous elements

$$
z_{1} \in A^{((1,0), 1)}, \quad z_{2} \in A^{((0,1), 1)}, \quad z_{1}^{*} \in A^{((-1,0),-1)}, \quad z_{2}^{*} \in A^{((0,-1),-1)},
$$

we observe that the non-vanishing components are as in (3.4). The formula for the $\star_{\theta}$-product on homogeneous elements follows directly from (2.9) and (2.8).

It follows from (3.4) that

$$
B=A^{\mathrm{co} H}=\bigoplus_{m \in \mathbb{Z}} A^{((m,-m), 0)} \subseteq A
$$

for the $*$-subalgebra of $H$-coinvariants.

Given any left $H$-comodule $(V, \rho)$, the vector space underlying the associated left $B$-module admits a decomposition

$$
A \square_{H} V=\bigoplus_{m, n \in \mathbb{Z}} A^{((m+n,-m), n)} \otimes V^{n},
$$

where

$$
V^{n}:=\left\{v \in V: \rho(v)=t^{n} \otimes v\right\} .
$$

By (3.5), the deformed left $B$-action of homogeneous elements $b \in A^{((m,-m), 0)} \subseteq B$ and $a \otimes v \in$ $A^{\left(\left(m^{\prime}+n^{\prime},-m^{\prime}\right), n^{\prime}\right)} \otimes V^{n^{\prime}} \subseteq A \square_{H} V$ is given by

$$
b \star_{\theta}(a \otimes v)=\left(b \star_{\theta} a\right) \otimes v=\mathrm{e}^{\pi \mathrm{i} \theta m n^{\prime}}(b a) \otimes v .
$$

Proposition 3.3. For every left $H$-comodule $(V, \rho)$, define a linear isomorphism

$$
L_{V}: A \square_{H} V \longrightarrow A_{\theta} \square_{H} V
$$

by setting

$$
L_{V}(a \otimes v)=\mathrm{e}^{\pi \mathrm{i} \theta m n} a \otimes v
$$

for all homogeneous elements $a \otimes v \in A^{((m+n,-m), n)} \otimes V^{n}$. This linear isomorphism is a left $B$-module isomorphism for $E_{A}(V)$ and $E_{A_{\theta}}(V)$. Moreover, the components (3.6) define a natural isomorphism $L: E_{A} \Longrightarrow E_{A_{\theta}}$ between the associated module functor for the classical Hopf fibration $\mathbb{S}^{3} \rightarrow \mathbb{S}^{2}$ and the one for the deformed Hopf fibration $\left(A_{\theta}, \delta\right)$.

Proof. For homogeneous elements $b \in A^{((m,-m), 0)} \subseteq B$ and $a \otimes v \in A^{\left(\left(m^{\prime}+n^{\prime},-m^{\prime}\right), n^{\prime}\right)} \otimes V^{n^{\prime}} \subseteq$ $A \square_{H} V$, we obtain

$$
L_{V}(b(a \otimes v))=\mathrm{e}^{\pi \mathrm{i} \theta\left(m+m^{\prime}\right) n^{\prime}}(b a) \otimes v,
$$

where $(b a) \otimes v \in A^{\left(\left(m+m^{\prime}+n^{\prime},-m-m^{\prime}\right), n^{\prime}\right)} \otimes V^{n^{\prime}}$, and

$$
b \star_{\theta} L_{V}(a \otimes v)=\mathrm{e}^{\pi \mathrm{i} \theta m^{\prime} n^{\prime}}\left(b \star_{\theta} a\right) \otimes v=\mathrm{e}^{\pi \mathrm{i} \theta m^{\prime} n^{\prime}} \mathrm{e}^{\pi \mathrm{i} \theta m n^{\prime}}(b a) \otimes v .
$$

These two terms coincide, hence $L_{V}$ is a left $B$-module isomorphism. Naturality is a straightforward check. 
Remark 3.4. We would like to emphasize that Proposition 3.3 is not a consequence of the general theory of 2-cocycle deformations from $[10,11]$ and [1]. In such a setting, it is convenient to observe that the $K$-coaction on $A$ induces to associated modules, i.e., $E_{A}:{ }^{H} \mathscr{M} \rightarrow{ }_{B}^{K} \mathscr{M}$ can be regarded as a functor to the category of left $K$-comodule left $B$-modules. Consequently, the deformed associated module functor $E_{A_{\theta}}:{ }^{H} \mathscr{M} \rightarrow{ }_{B_{\theta}}^{K_{\theta}} \mathscr{M}$ can be regarded as a functor to the category of left $K_{\theta}$-comodule left $B_{\theta}$-modules, where $K_{\theta}$ and $B_{\theta}$ are the 2-cocycle deformations of $K$ and $B$. (In our specific example of interest, we have that $K_{\theta}=K$ and $B_{\theta}=B$ (cf. Lemma 2.4), but we shall keep these labels to make the discussion below more transparent.) Using the 2-cocycle deformation functor from [11, Proposition 2.4] or [1, Proposition 2.25], which we shall denote by $\Sigma_{\theta}$, one obtains a commutative diagram

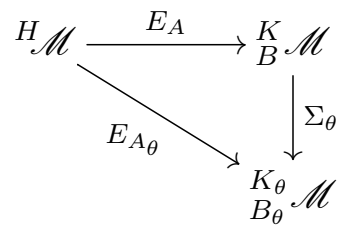

relating the associated module functor $E_{A_{\theta}}$ for the deformed Hopf fibration to the associated module functor $E_{A}$ for the classical one. In words, each deformed associated module $E_{A_{\theta}}(V)$ can be determined by applying the deformation functor to the classical associated module $E_{A}(V)$. For general 2-cocycle deformations that is all one can say.

For our special example given by the deformed Hopf fibration $A_{\theta}$, we have that $K_{\theta}=K$ and $B_{\theta}=B$ are undeformed, hence the deformed and the undeformed associated module functors $E_{A}, E_{A_{\theta}}:{ }^{H} \mathscr{M} \rightarrow{ }_{B}^{K} \mathscr{M}$ have the same target category. Proposition 3.3 proves that these two functors are already 'the same' (in the sense of naturally isomorphic) even if we do not use the deformation functor $\Sigma_{\theta}$. Hence, our natural isomorphism in Proposition 3.3 is more special and stronger than the results from the general theory of 2-cocycle deformations from [10, 11] and [1].

\section{The Atiyah sequence and connections}

\subsection{Differential geometry of principal comodule algebras}

Let us start with a brief review of some relevant concepts from noncommutative differential calculi, see, e.g., [30] and [8] for more details.

Definition 4.1. A (first-order) differential calculus on an algebra $A$ is a pair $\left(\Omega^{1}(A)\right.$, d) consisting of an $A$-bimodule $\Omega^{1}(A)$ and a linear map d: $A \rightarrow \Omega^{1}(A)$, such that

(i) $\mathrm{d}\left(a a^{\prime}\right)=a \mathrm{~d}\left(a^{\prime}\right)+\mathrm{d}(a) a^{\prime}$, for all $a, a^{\prime} \in A$,

(ii) $\Omega^{1}(A)=A \mathrm{~d}(A)=\left\{\sum_{j} a_{j} \mathrm{~d}\left(a_{j}^{\prime}\right): a_{j}, a_{j}^{\prime} \in A\right\}$.

We say that $\left(\Omega^{1}(A), \mathrm{d}\right)$ is connected if $\mathrm{d}(a)=0$ if and only if $a \in k \mathbf{1} \subseteq A$.

Every differential calculus is a quotient of the universal calculus $\left(\Gamma^{1}(A), \mathrm{d}_{\mathrm{u}}\right)$. Recall that the $A$-bimodule of universal 1-forms $\Gamma^{1}(A):=\operatorname{ker} \mu_{A} \subseteq A \otimes A$ is the kernel of the multiplication map and that the universal differential $\mathrm{d}_{\mathrm{u}}: A \rightarrow \Gamma^{1}(A)$ is defined as $\mathrm{d}_{\mathrm{u}}(a)=\mathbf{1} \otimes a-a \otimes \mathbf{1}$, for all $a \in A$. We find that $\left(\Gamma^{1}(A), \mathrm{d}_{\mathrm{u}}\right)$ is a connected differential calculus. Given any $A$-subbimodule $N \subseteq \Gamma^{1}(A)$, the $A$-bimodule $\Omega^{1}(A)=\Gamma^{1}(A) / N$ and

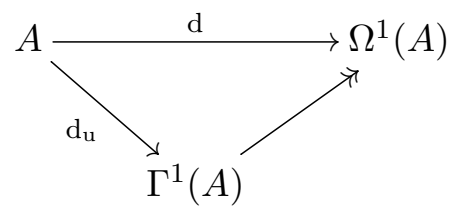


defines a differential calculus $\left(\Omega^{1}(A), \mathrm{d}\right)$. Vice versa, every differential calculus is of this form, see, e.g., [30, Proposition 6.1] for a proof.

The case where $H$ is a Hopf algebra was studied in detail in [35]. Any right ideal $Q \subseteq H^{+}$ of the augmentation ideal $H^{+}:=\operatorname{ker} \epsilon$ that is invariant under the right adjoint $H$-coaction ad: $H^{+} \rightarrow H^{+} \otimes H, h \mapsto h_{\underline{2}} \otimes S\left(h_{\underline{1}}\right) h_{\underline{3}}$, i.e., $\operatorname{ad}(Q) \subseteq Q \otimes H$, induces a bicovariant differential calculus on $H$. The corresponding $H$-subbimodule $N_{H} \subseteq \Gamma^{1}(H)$ is generated by the image of $Q \subseteq H^{+}$under the linear map $\kappa:=(S \otimes$ id $) \circ \Delta: H^{+} \rightarrow \Gamma^{1}(H)$. The quotient right $H$-comodule

$$
\mathfrak{h}_{Q}^{\vee}:=H^{+} / Q
$$

plays the role of the dual of the quantum Lie algebra of $H$ relative to $Q$. By construction, there exists a map $\kappa_{Q}$ that fits into the commutative diagram

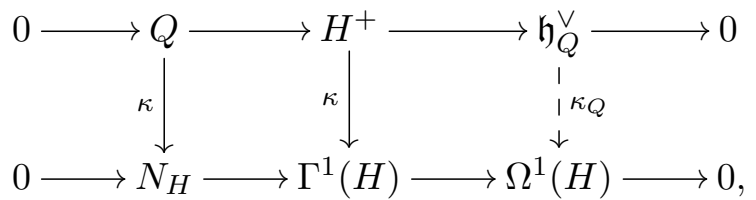

whose rows are short exact sequences. The resulting $H$-bimodule of 1 -forms $\Omega^{1}(H)=\Gamma^{1}(H) / N_{H}$ is freely generated by $\kappa_{Q}\left(\chi_{i}\right) \in \Omega^{1}(H)$, where $\left\{\chi_{i}\right\}$ is a basis of $\mathfrak{h}_{Q}^{\vee}$.

For a right $H$-comodule algebra $(A, \delta)$, it is natural to demand that the $A$-subbimodule $N \subseteq \Gamma^{1}(A)$ is invariant under the tensor product right $H$-coaction, i.e., $\delta(N) \subseteq N \otimes H$. More explicitly,

$$
\sum_{j}\left(a_{j}\right)_{\underline{0}} \otimes\left(a_{j}^{\prime}\right)_{\underline{0}} \otimes\left(a_{j}\right)_{\underline{1}}\left(a_{j}^{\prime}\right)_{\underline{1}} \in N \otimes H
$$

for all $\sum_{j} a_{j} \otimes a_{j}^{\prime} \in N$. The corresponding differential calculus $\left(\Omega^{1}(A), \mathrm{d}\right)$ then satisfies the property that $\Omega^{1}(A)$ is a right $H$-comodule $A$-bimodule and that $\mathrm{d}: A \rightarrow \Omega^{1}(A)$ is a right $H$-comodule morphism. Further, given any ad-invariant right ideal $Q \subseteq H^{+}$, we may require that $Q$ and $N$ are compatible in the sense that $\operatorname{ver}(N) \subseteq A \otimes Q$, where the (universal) vertical lift is defined as the linear map

$$
\begin{aligned}
\text { ver }:=\left(\mu_{A} \otimes \mathrm{id}\right) \circ(\mathrm{id} \otimes \delta): \Gamma^{1}(A) & \longrightarrow A \otimes H^{+}, \\
\sum_{j} a_{j} \otimes a_{j}^{\prime} & \longrightarrow \sum_{j} a_{j}\left(a_{j}^{\prime}\right)_{\underline{0}} \otimes\left(a_{j}^{\prime}\right)_{\underline{1}} .
\end{aligned}
$$

Observe that in this case, the (universal) vertical lift descends to a linear map $\overline{\mathrm{ver}}: \Omega^{1}(A) \rightarrow$ $A \otimes \mathfrak{h}_{Q}^{\vee}$ defined by the diagram

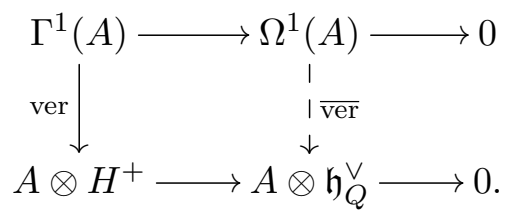

If moreover there is an equality $\operatorname{ver}(N)=A \otimes Q$, we say that the two differential calculi $\left(\Omega^{1}(A), \mathrm{d}\right)$ and $\left(\Omega^{1}(H), \mathrm{d}\right)$ are concordant. This definition is motivated by the result in [27] that the nonuniversal Atiyah sequence (see (4.3) below) associated to a principal $H$-comodule algebra $(A, \delta)$ is short exact if and only if the differential calculi on $A$ and $H$ are concordant. Loosely speaking, this means that in the case of concordant differential calculi one has an identification between 
the vertical vector fields on $A$ and the quantum Lie algebra of the structure Hopf algebra, which is in analogy to the fundamental vector field construction from classical differential geometry.

It is well-known, see, e.g., [15, Part VII, Proposition 6.6], that a right $H$-comodule algebra $(A, \delta)$ is a Hopf-Galois extension of the coinvariant subalgebra $B=A^{\operatorname{co} H}$ if and only if the (universal) Atiyah sequence

$$
0 \longrightarrow A \Gamma^{1}(B) A \longrightarrow \Gamma^{1}(A) \stackrel{\text { ver }}{\longrightarrow} A \otimes H^{+} \longrightarrow 0
$$

of right $H$-comodule left $A$-modules is short exact. In particular, for every principal $H$-comodule algebra $(A, \delta)$, the sequence $(4.2)$ is short exact. The right $H$-comodule $A$-bimodule

$$
A \Gamma^{1}(B) A:=\left\{\sum_{j} a_{j} b_{j} \otimes b_{j}^{\prime} a_{j}^{\prime}: a_{j}, a_{j}^{\prime} \in A, b_{j}, b_{j}^{\prime} \in B \text { with } \sum_{j} a_{j} b_{j} b_{j}^{\prime} a_{j}^{\prime}=0\right\}
$$

is called the module of universal horizontal 1-forms. Let us also recall that the right $H$-coactions on both $A \Gamma^{1}(B) A$ and $\Gamma^{1}(A)$ are induced by the tensor product coaction on $A \otimes A$. As well, the right $H$-coaction on $A \otimes H^{+}$is the tensor product coaction with $H^{+}$endowed with the right adjoint $H$-coaction ad: $h \mapsto h_{\underline{2}} \otimes S\left(h_{\underline{1}}\right) h_{\underline{3}}$.

Definition 4.2. A connection with respect to the universal differential calculus on a principal $H$-comodule algebra $(A, \delta)$ is a right $H$-comodule left $A$-module splitting s: $A \otimes H^{+} \rightarrow \Gamma^{1}(A)$ of (4.2), i.e., ver $\circ \mathrm{s}=\mathrm{id}$. Every connection $\mathrm{s}$ is fully determined by its connection form

$$
\omega: H^{+} \longrightarrow \Gamma^{1}(A), \quad h \longmapsto \mathbf{s}\left(\mathbf{1}_{A} \otimes h\right) .
$$

Associated to a connection s: $A \otimes H^{+} \rightarrow \Gamma^{1}(A)$, or equivalently to its connection form $\omega: H^{+} \rightarrow \Gamma^{1}(A)$, is its covariant derivative

$$
D:=(\mathrm{id}-\mathrm{s} \circ \text { ver }) \circ \mathrm{d}_{\mathrm{u}}: A \longrightarrow A \Gamma^{1}(B) A, \quad a \longmapsto \mathrm{d}_{\mathrm{u}}(a)-a_{\underline{0}} \omega\left(a_{\underline{1}}-\epsilon\left(a_{\underline{1}}\right) \mathbf{1}_{H}\right) .
$$

A connection is called strong provided that $D(A) \subseteq \Gamma^{1}(B) A$. Strong connections are in one-toone correspondence with maps $\ell: H \rightarrow A \otimes A$ satisfying the conditions (2.1) in Definition 2.1, see, e.g., [24] or [14]. A covariant derivative $D$ of a strong connection is a connection on the left $B$-module $A$, in the sense of [23]. Every strong connection defines a connection, for the universal calculus, on the associated left $B$-module $E_{A}(V)=A \square_{H} V$ via

$$
\nabla: E_{A}(V) \longrightarrow \Gamma^{1}(B) \otimes_{B} E_{A}(V), \quad a \otimes v \longmapsto D(a) \otimes v .
$$

It was shown in [27] that for concordant differential calculi $\left(\Omega^{1}(A), \mathrm{d}\right)$ and $\left(\Omega^{1}(H)\right.$, d), i.e., $\operatorname{ver}(N)=A \otimes Q$, exactness of the universal Atiyah sequence (4.2) implies exactness of the induced sequence

$$
0 \longrightarrow \Omega^{1}(A)_{\text {hor }} \longrightarrow \Omega^{1}(A) \stackrel{\overline{\mathrm{ver}}}{\longrightarrow} A \otimes \mathfrak{h}_{Q}^{\vee} \longrightarrow 0
$$

of right $H$-comodule left $A$-modules. The module of horizontal 1-forms is defined by

$$
\Omega^{1}(A)_{\text {hor }}:=\frac{A \Gamma^{1}(B) A}{A \Gamma^{1}(B) A \cap N}=A \Omega^{1}(B) A,
$$

where $\left(\Omega^{1}(B), \mathrm{d}\right)$ is the differential calculus on $B$ that is determined by the $B$-subbimodule $N_{B}:=\left(\operatorname{ker} \mu_{B}\right) \cap N \subseteq \Gamma^{1}(B)$. Equivalently, this differential calculus may by obtained from $\left(\Omega^{1}(A), \mathrm{d}\right)$ as a restriction, i.e., $\Omega^{1}(B)=B \mathrm{~d}(B) \subseteq \Omega^{1}(A)$, see, e.g., [8, Chapter 5]. The short exact sequence (4.3) will be referred to as the Atiyah sequence for the concordant differential calculi $\left(\Omega^{1}(A), \mathrm{d}\right)$ and $\left(\Omega^{1}(H), \mathrm{d}\right)$. 
Definition 4.3. A connection with respect to concordant differential calculi $\left(\Omega^{1}(A), \mathrm{d}\right)$ and $\left(\Omega^{1}(H), \mathrm{d}\right)$ on a principal $H$-comodule algebra $(A, \delta)$ is a right $H$-comodule left $A$-module splitting $\overline{\mathrm{s}}: A \otimes \mathfrak{h}_{Q}^{\vee} \rightarrow \Omega^{1}(A)$ of the Atiyah sequence (4.3), i.e., $\overline{\mathrm{ver}} \circ \overline{\mathrm{s}}=\mathrm{id}$. Every connection $\overline{\mathrm{s}}$ is fully determined by its connection form

$$
\bar{\omega}: \mathfrak{h}_{Q}^{\vee} \longrightarrow \Omega^{1}(A), \quad h \longmapsto \overline{\mathbf{s}}\left(\mathbf{1}_{A} \otimes h\right) .
$$

Similarly to the case of the universal calculus above, associated to a connection $\overline{\mathrm{s}}: A \otimes \mathfrak{h}_{Q}^{\vee} \rightarrow$ $\Omega^{1}(A)$, or equivalently to its connection form $\bar{\omega}: \mathfrak{h}_{Q}^{\vee} \rightarrow \Omega^{1}(A)$, is its covariant derivative

$$
\bar{D}:=(\mathrm{id}-\overline{\mathrm{s}} \circ \overline{\mathrm{ver}}) \circ \mathrm{d}: A \longrightarrow \Omega_{\mathrm{hor}}^{1}(A), \quad a \longmapsto \mathrm{d}(a)-a_{\underline{0}} \bar{\omega}\left(a_{\underline{1}}-\epsilon\left(a_{\underline{1}}\right) \mathbf{1}_{H}\right) .
$$

A connection is said to be strong provided that $\bar{D}(A) \subseteq \Omega^{1}(B) A$. Every strong connection defines a connection for the differential calculus $\left(\Omega^{1}(B), \mathrm{d}\right)$ on the associated left $B$-module $E_{A}(V)=A \square_{H} V$ via

$$
\bar{\nabla}: E_{A}(V) \longrightarrow \Omega^{1}(B) \otimes_{B} E_{A}(V), \quad a \otimes v \longmapsto \bar{D}(a) \otimes v .
$$

\subsection{The universal Atiyah sequence for Hopf fibrations}

Consider the $(K, H)$-bicomodule algebra $\left(A_{\theta}, \rho, \delta\right)$ from Section 2.3 which describes the deformed Hopf fibration. As the underlying right $H$-comodule algebra $\left(A_{\theta}, \delta\right)$ is a principal comodule algebra (cf. Proposition 2.5), we obtain from (4.2) the corresponding (universal) Atiyah sequence

$$
0 \longrightarrow A_{\theta} \Gamma^{1}(B) A_{\theta} \longrightarrow \Gamma^{1}\left(A_{\theta}\right) \stackrel{\text { ver }_{\theta}}{\longrightarrow} A_{\theta} \otimes H^{+} \longrightarrow 0 .
$$

Let us emphasize that $B=A_{\theta}^{\operatorname{co} H} \subseteq A_{\theta}$ is the algebra of functions on the classical 2-sphere (cf. Lemma 2.4) and that the vertical lift (4.1), in the present case, involves the $\star_{\theta}$-product of $A_{\theta}$, i.e.,

$$
\operatorname{ver}_{\theta}: \Gamma^{1}\left(A_{\theta}\right) \longrightarrow A_{\theta} \otimes H^{+}, \quad \sum_{j} a_{j} \otimes a_{j}^{\prime} \longmapsto \sum_{j}\left(a_{j} \star_{\theta}\left(a_{j}^{\prime}\right)_{0}\right) \otimes\left(a_{j}^{\prime}\right)_{\underline{1}} .
$$

Note that (4.6) is a short exact sequence of $(K, H)$-bicomodule left $A_{\theta}$-modules. Setting the deformation parameter $\theta=0$, we obtain the (universal) Atiyah sequence of the classical Hopf fibration $\mathbb{S}^{3} \rightarrow \mathbb{S}^{2}$

$$
0 \longrightarrow A \Gamma^{1}(B) A \longrightarrow \Gamma^{1}(A) \stackrel{\text { ver }}{\longrightarrow} A \otimes H^{+} \longrightarrow 0 .
$$

This is a short exact sequence of $(K, H)$-bicomodule left $A$-modules. Utilizing the 2-cocycle deformation functor from [11, Proposition 2.4] or [1, Proposition 2.25(ii)], we obtain a short exact sequence of $(K, H)$-bicomodule left $A_{\theta}$-modules

$$
0 \longrightarrow\left(A \Gamma^{1}(B) A\right)_{\theta} \longrightarrow \Gamma^{1}(A)_{\theta} \stackrel{\text { ver }}{\longrightarrow}\left(A \otimes H^{+}\right)_{\theta} \longrightarrow 0 .
$$

As a sequence of $(K, H)$-bicomodules, (4.8) coincides with (4.7). The left $A_{\theta}$-module structures on the objects in (4.8) are obtained by the following construction: Given any $(K, H)$-bicomodule left $A$-module $M$, the deformed left $A_{\theta}$-action on the $(K, H)$-bicomodule left $A_{\theta}$-module $M_{\theta}$ is

$$
a \star_{\theta} m:=\sigma_{\theta}\left(a_{\underline{-1}} \otimes m_{\underline{-1}}\right) a_{\underline{\underline{0}}} m_{\underline{\underline{0}}},
$$

for all $a \in A_{\theta}$ and $m \in M_{\theta}$. 
Proposition 4.4. Consider the $(K, H)$-bicomodule isomorphism

$$
\varphi_{\theta}: \quad A_{\theta} \otimes A_{\theta} \longrightarrow(A \otimes A)_{\theta}, \quad a \otimes a^{\prime} \longmapsto \sigma_{\theta}\left(a_{\underline{-1}} \otimes a_{\underline{-1}}^{\prime}\right) a_{\underline{0}} \otimes a_{\underline{\underline{ }}}^{\prime} .
$$

Then

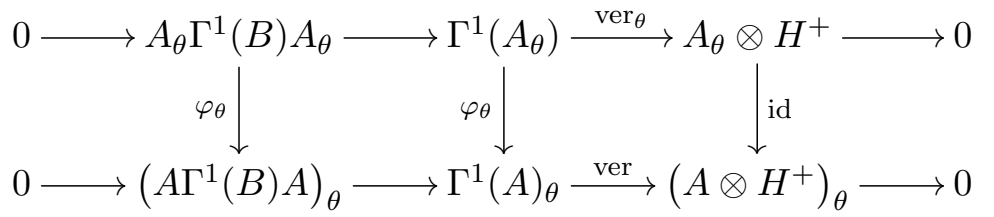

is an isomorphism of short exact sequences of $(K, H)$-bicomodule left $A_{\theta}$-modules.

Proof. Note that (4.9) relates the deformed and undeformed product via $\mu_{A_{\theta}}=\mu_{A} \circ \varphi_{\theta}$. As a consequence, it restricts to the middle vertical arrow in (4.10). The 2-cocycle property of $\sigma_{\theta}$ implies that the middle vertical arrow is a $(K, H)$-bicomodule left $A_{\theta}$-module isomorphism. From (2.12) we find that $\varphi_{\theta}$ acts as the identity on $\Gamma^{1}(B) \subseteq B \otimes B \subseteq A_{\theta} \otimes A_{\theta}$. Together with the previous result, this implies that the left vertical arrow in (4.10) has the claimed domain and codomain and that it is a $(K, H)$-bicomodule left $A_{\theta}$-module isomorphism. The left square commutes by construction.

The right vertical arrow in $(4.10)$ is a $(K, H)$-bicomodule left $A_{\theta}$-module isomorphism because the $K$-coaction on $H^{+}$is trivial. We see directly that the right square commutes by

$$
\begin{aligned}
\operatorname{ver} \circ \varphi_{\theta}\left(\sum_{j} a_{j} \otimes a_{j}^{\prime}\right) & =\sum_{j} \sigma_{\theta}\left(\left(a_{j}\right)_{\underline{-1}} \otimes\left(a_{j}^{\prime}\right)_{\underline{-1}}\right)\left(a_{j}\right)_{\underline{0}}\left(a_{j}^{\prime}\right)_{\underline{0}_{\underline{0}}} \otimes\left(a_{j}^{\prime}\right)_{\underline{0}_{\underline{1}}} \\
& =\sum_{j} \sigma_{\theta}\left(\left(a_{j}\right)_{\underline{-1}} \otimes\left(a_{j}^{\prime} \underline{\underline{\underline{0}}}_{\underline{-1}}\right)\left(a_{j}\right)_{\underline{\underline{0}}}\left(a_{j}^{\prime}\right)_{\underline{0}_{\underline{0}}} \otimes\left(a_{j}^{\prime}\right)_{\underline{1}}\right. \\
& =\sum_{j} a_{j} \star_{\theta}\left(a_{j}^{\prime}\right)_{\underline{0}} \otimes\left(a_{j}^{\prime}\right)_{\underline{1}}=\operatorname{ver}_{\theta}\left(\sum_{j} a_{j} \otimes a_{j}^{\prime}\right),
\end{aligned}
$$

for all $\sum_{j} a_{j} \otimes a_{j}^{\prime} \in \Gamma^{1}\left(A_{\theta}\right)$, where in the second step we used (2.6).

\subsection{The Atiyah sequence for Kähler forms}

For a commutative algebra $A$, the product map $\mu_{A}: A \otimes A \rightarrow A$ is an algebra homomorphism when $A \otimes A$ is endowed with the tensor algebra structure $\left(a \otimes a^{\prime}\right)\left(\widetilde{a} \otimes \widetilde{a}^{\prime}\right):=(a \widetilde{a}) \otimes\left(a^{\prime} \widetilde{a}^{\prime}\right)$. This implies that $\operatorname{ker} \mu_{A} \subseteq A \otimes A$ is an ideal. Recall that the module of Kähler 1-forms on $A$ is defined as the quotient $A$-bimodule

$$
\Omega^{1}(A):=\frac{\operatorname{ker} \mu_{A}}{\left(\operatorname{ker} \mu_{A}\right)^{2}}=\frac{\Gamma^{1}(A)}{\left(\operatorname{ker} \mu_{A}\right)^{2}} .
$$

The Kähler differential d: $A \rightarrow \Omega^{1}(A)$ is the composition of the universal differential $\mathrm{d}_{\mathrm{u}}: A \rightarrow$ $\Gamma^{1}(A)$ and the quotient map $\Gamma^{1}(A) \rightarrow \Omega^{1}(A)$. In other words, $\left(\Omega^{1}(A), \mathrm{d}\right)$ is the first-order differential calculus presented by the quotient of the universal calculus $\left(\Gamma^{1}(A), \mathrm{d}_{\mathrm{u}}\right)$ by the $A$ subbimodule $N:=\left(\operatorname{ker} \mu_{A}\right)^{2} \subseteq \Gamma^{1}(A)$.

For a commutative Hopf algebra $H$, the Kähler differential calculus on $H$ is bicovariant and it corresponds to the ad-invariant right ideal $Q=\left(H^{+}\right)^{2}$, where $H^{+}=\operatorname{ker} \epsilon$. Given further a commutative principal $H$-comodule algebra $(A, \delta)$, with coinvariants $B=A^{\mathrm{co} H}$, both $\Gamma^{1}(A)=$ ker $\mu_{A} \subseteq A \otimes A$ and $A \otimes H^{+} \subseteq A \otimes H$ are ideals with respect to the tensor algebra structures. It is easily checked that the vertical lift ver: $\Gamma^{1}(A) \rightarrow A \otimes H^{+}$is an algebra homomorphism, 
hence it maps $\left(\operatorname{ker} \mu_{A}\right)^{2}$ to $\left(A \otimes H^{+}\right)^{2}=A \otimes\left(H^{+}\right)^{2}$. Because the latter map is surjective, the Kähler differential calculi on $A$ and $H$ are concordant. Hence, there is a corresponding short exact Atiyah sequence (4.3) for Kähler forms in which

$$
\Omega_{\mathrm{hor}}^{1}(A):=\frac{A \Gamma^{1}(B) A}{\left(A \Gamma^{1}(B) A\right) \cap\left(\operatorname{ker} \mu_{A}\right)^{2}}=A \Omega^{1}(B) A, \quad \mathfrak{h}^{\vee}:=\mathfrak{h}_{Q}^{\vee}=\frac{H^{+}}{\left(H^{+}\right)^{2}},
$$

and $\Omega^{1}(B)=\Gamma^{1}(B) / N_{B}$ is determined by $N_{B}=\left(\operatorname{ker} \mu_{A}\right)^{2} \cap \Gamma^{1}(B) \subseteq \Gamma^{1}(B)$.

It might be worth pointing out that, in general, $\Omega^{1}(B)$ defined above by the restriction of the Kähler 1-forms on $A$ is not necessarily the module of Kähler 1-forms on $B$.

Example 4.5. Let $k$ be a field of characteristic $p$ and $A=k\left[x, x^{-1}\right]$ the commutative algebra of Laurent polynomials in $x$. Further, let $H=k \mathbb{Z}_{p}$ be the group Hopf algebra of the cyclic group of order $p$. The latter may be presented as the commutative algebra $H=k[\xi] /\left(\mathbf{1}-\xi^{p}\right)$, equipped with the coproduct $\Delta(\xi)=\xi \otimes \xi$, counit $\epsilon(\xi)=1$ and antipode $S(\xi)=\xi^{-1}$. (By $\xi^{-1} \in H$ we mean the element represented by $\xi^{p-1} \in k[\xi]$.) We endow $A$ with the structure of a right $H$-comodule algebra by defining $\delta(x)=x \otimes \xi$ and $\delta\left(x^{-1}\right)=x^{-1} \otimes \xi^{-1}$. The $H$-coinvariant subalgebra $B=k\left[x^{p}, x^{-p}\right] \subseteq k\left[x, x^{-1}\right]=A$ is the algebra of Laurent polynomials in $x^{p}$. We further observe that $(A, \delta)$ is a principal comodule algebra with strong connection $\ell: H \rightarrow A \otimes A$ defined by

$$
\ell\left(\xi^{n}\right)=x^{-n} \otimes x^{n}, \quad \text { for } \quad n=0, \ldots, p-1 .
$$

Because $A=k\left[x, x^{-1}\right] \cong k[x, y] /(x y-\mathbf{1})$ admits a finite presentation by two generators $x$ and $y$, the corresponding module of Kähler 1-forms is isomorphic to the quotient $\Omega^{1}(A) \cong$ $(A \mathrm{~d} x \oplus A \mathrm{~d} y) /\langle y \mathrm{~d} x+x \mathrm{~d} y\rangle$, see, e.g., [25, Section 16.1]. The generator $\mathrm{d} y$ can be eliminated by the relation $\mathrm{d} y=-y^{2} \mathrm{~d} x$, which implies that $\Omega^{1}(A)=A \mathrm{~d} x$. (Note that $\mathrm{d} x^{-1}=-x^{-2} \mathrm{~d} x$ in this calculus.) Restricting the Kähler 1-forms $\Omega^{1}(A)$ to $B=k\left[x^{p}, x^{-p}\right] \subseteq k\left[x, x^{-1}\right]=A$ defines the trivial differential calculus $\Omega^{1}(B)=0$. This follows as $k$ is a field of characteristic $p$, by hypothesis, and hence $\mathrm{d}\left(x^{p}\right)=p x^{p-1} \mathrm{~d} x=0$. On the other hand, the Kähler differential calculus on $B$ is non-trivial. ${ }^{1}$

In view of the above comment and example, it is useful to observe the following lemma.

Lemma 4.6. Let $A=k\left[x_{1}, \ldots, x_{n}\right] / J_{A}$ be a finitely generated algebra and $B \subseteq A$ the subalgebra generated by $X_{1}, \ldots, X_{m} \in k\left[x_{1}, \ldots, x_{n}\right]$. If the set $\left\{\mathrm{d}_{x}\left(X_{1}\right), \ldots, \mathrm{d}_{x}\left(X_{m}\right)\right\}$ is free in the module underlying the Kähler differential calculus $\left(\Omega^{1}\left(k\left[x_{1}, \ldots, x_{n}\right]\right), \mathrm{d}_{x}\right)$ on the algebra $k\left[x_{1}, \ldots, x_{n}\right]$, then the Kähler differential calculus $\left(\Omega^{1}(B), \mathrm{d}_{B}\right)$ on $B$ is isomorphic to the restriction $\left(B \mathrm{~d}_{A}(B), \mathrm{d}_{A}\right) \subseteq\left(\Omega^{1}(A), \mathrm{d}_{A}\right)$ to $B \subseteq A$ of the Kähler differential calculus on $A$.

Proof. Recall from [25, Proposition 16.1] that $\Omega^{1}\left(k\left[x_{1}, \ldots, x_{n}\right]\right)$ and $\Omega^{1}\left(k\left[X_{1}, \ldots, X_{m}\right]\right)$ are free modules. By our hypothesis that $\left\{\mathrm{d}_{x}\left(X_{1}\right), \ldots, \mathrm{d}_{x}\left(X_{m}\right)\right\}$ is free in $\Omega^{1}\left(k\left[x_{1}, \ldots, x_{n}\right]\right)$, it follows that the map

$$
\Omega^{1}\left(k\left[X_{1}, \ldots, X_{m}\right]\right) \longrightarrow \Omega^{1}\left(k\left[x_{1}, \ldots, x_{n}\right]\right), \quad \sum_{j} f_{j} \mathrm{~d}_{X}\left(X_{j}\right) \longmapsto \sum_{j} f_{j} \mathrm{~d}_{x}\left(X_{j}\right)
$$

is injective and hence it defines an isomorphism

$$
\Omega^{1}\left(k\left[X_{1}, \ldots, X_{m}\right]\right) \cong k\left[X_{1}, \ldots, X_{m}\right] \mathrm{d}_{x}\left(k\left[X_{1}, \ldots, X_{m}\right]\right) .
$$

As $\Omega^{1}(A)$ is given by the quotient of $\Omega^{1}\left(k\left[x_{1}, \ldots, x_{n}\right]\right)$ by the ideal $\left(J_{A}, \mathrm{~d}_{x} J_{A}\right)$ generated by $J_{A}$ and $\mathrm{d}_{x} J_{A}$, and likewise $\Omega^{1}(B)=\Omega^{1}\left(k\left[X_{1}, \ldots, X_{m}\right]\right) /\left(J_{B}, \mathrm{~d}_{X} J_{B}\right)$ with $J_{B}:=J_{A} \cap k\left[X_{1}, \ldots, X_{m}\right]$,

\footnotetext{
${ }^{1}$ We are grateful to Christian Lomp for his comment, which led us to Example 4.5.
} 
see, e.g., [25, Section 16.1], the required isomorphism is induced by the following diagram with exact rows

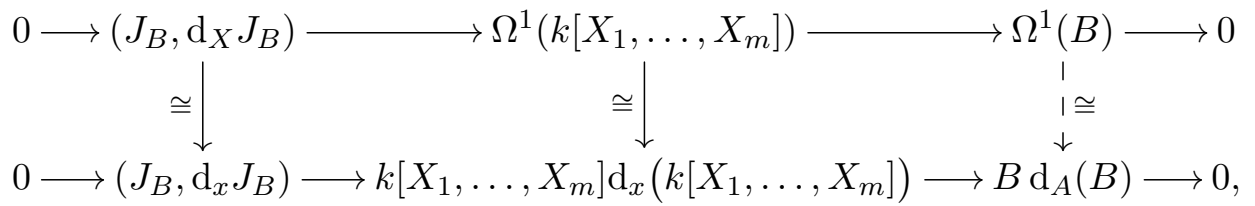

where the solid vertical arrows arise from (4.11).

Let us consider now the Atiyah sequence (4.3) for the classical Hopf fibration $\mathbb{S}^{3} \rightarrow \mathbb{S}^{2}$ and the Kähler differential calculi on $A=\mathcal{O}\left(\mathbb{S}^{3}\right)$ and $H=\mathcal{O}(U(1))$, i.e.,

$$
0 \longrightarrow \Omega_{\mathrm{hor}}^{1}(A) \longrightarrow \Omega^{1}(A) \stackrel{\overline{\mathrm{ver}}}{\longrightarrow} A \otimes \mathfrak{h}^{\vee} \longrightarrow 0 .
$$

The vector space $\mathfrak{h}^{\vee}=H^{+} /\left(H^{+}\right)^{2}$ is the algebraic cotangent space of $U(1)$ at the unit element. Its dual is the vector space underlying the Lie algebra of $U(1)$, i.e., the vector space $\mathfrak{h}:=\operatorname{Der}_{\epsilon}(H)$ of derivations relative to $\epsilon: H \rightarrow \mathbb{C}$. Recall that $X \in \operatorname{Der}_{\epsilon}(H)$ is a linear map $X: H \rightarrow \mathbb{C}$ such that $X\left(h h^{\prime}\right)=X(h) \epsilon\left(h^{\prime}\right)+\epsilon(h) X\left(h^{\prime}\right)$, for all $h, h^{\prime} \in H$. It is easily checked that $\mathfrak{h}=\operatorname{Der}_{\epsilon}(H) \cong \mathbb{C}$ is one-dimensional. Furthermore, since the generators $z=2 z_{1} z_{2}^{*}, z^{*}=2 z_{1}^{*} z_{2}$ and $x=z_{1}^{*} z_{1}-z_{2}^{*} z_{2}$ of $B=A^{\mathrm{co} H} \cong \mathcal{O}\left(\mathbb{S}^{2}\right)$ (cf. Lemma 2.2) satisfy the conditions of Lemma 4.6, the horizontal forms in $(4.12)$ are

$$
\Omega_{\mathrm{hor}}^{1}(A)=A \Omega^{1}(B) A=A \Omega^{1}(B)=\Omega^{1}(B) A, \quad \text { with } \quad \Omega^{1}(B)=\frac{\operatorname{ker} \mu_{B}}{\left(\operatorname{ker} \mu_{B}\right)^{2}} .
$$

As $(A, \delta, \rho)$ is a $(K, H)$-bicomodule algebra, it follows that (4.12) is a short exact sequence of $(K, H)$-bicomodule left $A$-modules. Applying the 2-cocycle deformation functor from [11, Proposition 2.4] or [1, Proposition 2.25(ii)], we obtain the short exact sequence of $(K, H)$ bicomodule left $A_{\theta}$-modules

$$
0 \longrightarrow \Omega_{\text {hor }}^{1}(A)_{\theta} \longrightarrow \Omega^{1}(A)_{\theta} \stackrel{\overline{\mathrm{ver}}}{\longrightarrow}\left(A \otimes \mathfrak{h}^{\vee}\right)_{\theta} \longrightarrow 0 .
$$

A similar construction applies to the deformed Hopf fibration described by the $(K, H)$ bicomodule algebra $\left(A_{\theta}, \rho, \delta\right)$ from Section 2.3. The key point is that $A_{\theta}$ is braided commutative (see, e.g., [4]) in the sense of

$$
a \star_{\theta} a^{\prime}=R_{\theta}\left(a_{\underline{-1}}^{\prime} \otimes a_{\underline{-1}}\right) a_{\underline{0}}^{\prime} \star_{\theta} a_{\underline{0}},
$$

for all $a, a^{\prime} \in A_{\theta}$, with cotriangular structure $R_{\theta}: K \otimes K \rightarrow \mathbb{C}$ given by

$$
R_{\theta}\left(t_{\mathbf{m}} \otimes t_{\mathbf{m}^{\prime}}\right)=\sigma_{\theta}\left(t_{\mathbf{m}} \otimes t_{\mathbf{m}^{\prime}}\right)^{-2}=\exp \left(-2 \pi \mathbf{i m}^{\mathrm{T}} \Theta \mathbf{m}^{\prime}\right) .
$$

The product map $\mu_{A_{\theta}}: A_{\theta} \otimes A_{\theta} \rightarrow A_{\theta}$ is an algebra homomorphism when $A_{\theta} \otimes A_{\theta}$ is endowed with the braided tensor algebra structure $\left(a \otimes a^{\prime}\right)\left(\widetilde{a} \otimes \widetilde{a}^{\prime}\right):=R_{\theta}\left(\widetilde{a}_{-1} \otimes a^{\prime}{ }_{-1}\right)\left(a \star_{\theta} \widetilde{a}_{\underline{0}}\right) \otimes\left(a_{\underline{0}}^{\prime} \star_{\theta} \widetilde{a}^{\prime}\right)$.

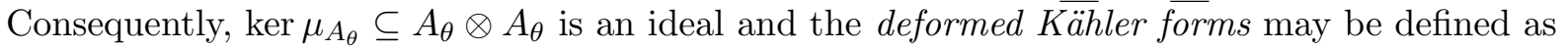

$$
\Omega^{1}\left(A_{\theta}\right):=\frac{\operatorname{ker} \mu_{A_{\theta}}}{\left(\operatorname{ker} \mu_{A_{\theta}}\right)^{2}} .
$$

The vertical lift in (4.6) is an algebra homomorphism with respect to the braided tensor algebra structures. Hence, it maps $\left(\operatorname{ker} \mu_{A_{\theta}}\right)^{2}$ to $\left(A_{\theta} \otimes H^{+}\right)^{2}=A_{\theta} \otimes\left(H^{+}\right)^{2}$. In analogy to (4.12), we obtain the quotient short exact sequence

$$
0 \longrightarrow \Omega_{\text {hor }}^{1}\left(A_{\theta}\right) \longrightarrow \Omega^{1}\left(A_{\theta}\right) \stackrel{\overline{\operatorname{ver}}_{\theta}}{\longrightarrow} A_{\theta} \otimes \mathfrak{h}^{\vee} \longrightarrow 0 .
$$


Proposition 4.7. The isomorphism $\varphi_{\theta}: A_{\theta} \otimes A_{\theta} \rightarrow(A \otimes A)_{\theta}$ given in (4.9) descends to the $(K, H)$-bicomodule left $A_{\theta}$-module isomorphism

$$
\bar{\varphi}_{\theta}: \Omega^{1}\left(A_{\theta}\right) \longrightarrow \Omega^{1}(A)_{\theta}, \quad a \mathrm{~d}_{\theta} a^{\prime} \longmapsto a \star_{\theta} \mathrm{d} a^{\prime} .
$$

This defines an isomorphism of short exact sequences

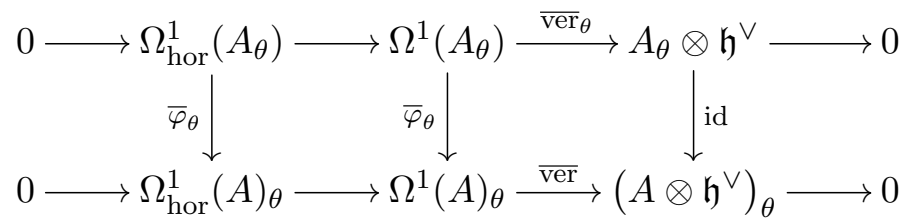

between (4.16) and (4.14).

Proof. Using the 2-cocycle property (2.7) of $\sigma_{\theta}$, it can be shown that the map $\varphi_{\theta}: A_{\theta} \otimes A_{\theta} \rightarrow$ $(A \otimes A)_{\theta}$ given in (4.9) is an algebra isomorphism with respect to the following algebra structures: As above, the domain $A_{\theta} \otimes A_{\theta}$ is endowed with the braided tensor algebra structure, i.e.,

$$
\left(a \otimes a^{\prime}\right)\left(\widetilde{a} \otimes \widetilde{a}^{\prime}\right):=R_{\theta}\left(\widetilde{a}_{\underline{-1}} \otimes a_{\underline{-1}}^{\prime}\right)\left(a \star_{\theta} \widetilde{a}_{\underline{0}}\right) \otimes\left(a_{\underline{0}}^{\prime} \star_{\theta} \widetilde{a}^{\prime}\right) .
$$

The codomain $(A \otimes A)_{\theta}$ is endowed with the 2-cocycle deformation of the usual tensor algebra structure on $A \otimes A$, i.e.,

$$
\left(a \otimes a^{\prime}\right) \star_{\theta}\left(\widetilde{a} \otimes \widetilde{a}^{\prime}\right):=\sigma_{\theta}\left(a_{\underline{-1}} a^{\prime}{ }_{-1} \otimes \widetilde{a}_{\underline{-1}} \widetilde{a}_{\underline{-1}}^{\prime}\right)\left(a_{\underline{0}} \widetilde{a}_{0}\right) \otimes\left(a_{\underline{0}}^{\prime} \widetilde{a}_{\underline{0}}^{\prime}\right) .
$$

As a consequence, $\varphi_{\theta}$ restricts to an isomorphism $\varphi_{\theta}:\left(\operatorname{ker} \mu_{A_{\theta}}\right)^{2} \rightarrow\left(\operatorname{ker} \mu_{A}\right)_{\theta}^{2}$, which implies that it descends to the claimed isomorphism $\bar{\varphi}_{\theta}$ between the quotient modules. The explicit expression for $\bar{\varphi}_{\theta}$ given in (4.17) follows from the computation

$$
\begin{aligned}
\bar{\varphi}_{\theta}\left(a \mathrm{~d}_{\theta} a^{\prime}\right) & =\varphi_{\theta}\left(a \otimes a^{\prime}-a \star_{\theta} a^{\prime} \otimes \mathbf{1}\right)=\sigma_{\theta}\left(a_{\underline{-1}} \otimes a_{\underline{-1}}^{\prime}\right)\left(a_{\underline{0}} \otimes a_{\underline{0}}^{\prime}-a_{\underline{0}} a_{\underline{0}}^{\prime} \otimes \mathbf{1}\right) \\
& =\sigma_{\theta}\left(a_{\underline{-1}} \otimes a_{\underline{-1}}^{\prime}\right) a_{\underline{0}} \mathrm{~d}\left(a_{\underline{0}}^{\prime}\right)=a \star_{\theta} \mathrm{d} a^{\prime},
\end{aligned}
$$

where in the second step we used (4.9) and (2.9), and in the last step we used that $\mathrm{d}$ is a $K$ comodule map. The statement about short exact sequences follows by using also Proposition 4.4 .

Corollary 4.8. $\Omega_{\text {hor }}^{1}\left(A_{\theta}\right)=A_{\theta} \Omega^{1}(B) A_{\theta}=A_{\theta} \Omega^{1}(B)=\Omega^{1}(B) A_{\theta}$ with $\Omega^{1}(B)$ the Kähler 1-forms on $B=A_{\theta}^{\operatorname{coH}} \subseteq A_{\theta}$.

Proof. By (4.13) and invertibility of the 2-cocycle $\sigma_{\theta}$, it follows that

$$
\Omega_{\mathrm{hor}}^{1}(A)_{\theta}=A_{\theta} \star_{\theta} \Omega^{1}(B) \star_{\theta} A_{\theta}=A_{\theta} \star_{\theta} \Omega^{1}(B)=\Omega^{1}(B) \star_{\theta} A_{\theta},
$$

where $\star_{\theta}$ denotes the deformed left and right $A_{\theta}$-module structures on $\Omega^{1}(A)_{\theta}$. Our claim then follows from the isomorphism $\bar{\varphi}_{\theta}$ given in (4.17), because it maps bijectively between $a\left(\mathrm{~d}_{\theta} b\right) a^{\prime} \in A_{\theta} \Omega^{1}(B) A_{\theta} \subseteq \Omega^{1}\left(A_{\theta}\right)$ and $a \star_{\theta}(\mathrm{d} b) \star_{\theta} a^{\prime} \in A_{\theta} \star_{\theta} \Omega^{1}(B) \star_{\theta} A_{\theta} \subseteq \Omega^{1}(A)_{\theta}$.

\subsection{Connections}

The aim of this section is to characterize connections with respect to the Kähler differential calculi for both the classical Hopf fibration $\mathbb{S}^{3} \rightarrow \mathbb{S}^{2}$ and the deformed Hopf fibration. It will be shown that they are equivalent in a suitable sense. 
We first consider the classical Hopf fibration $(A, \delta)$ from Section 2.2. By Definition 4.3, the set of connections is the set of splittings of (4.12) or equivalently the set of connection forms

$$
\operatorname{Con}(A, \delta):=\left\{\bar{\omega} \in \operatorname{Hom}^{H}\left(\mathfrak{h}^{\vee}, \Omega^{1}(A)\right): \overline{\operatorname{ver}} \circ \bar{\omega}=\mathbf{1} \otimes \mathrm{id}\right\}
$$

where $\operatorname{Hom}^{H}$ denotes the set of right $H$-comodule morphisms. Let us recall that $\mathfrak{h}=\operatorname{Der}_{\epsilon}(H) \cong$ $\mathbb{C}$ is 1-dimensional and choose a basis $X \in \mathfrak{h}$, e.g., the linear map

$$
X: H \longrightarrow \mathbb{C}, \quad t^{n} \longmapsto n .
$$

Let $\chi \in \mathfrak{h}^{\vee}$ be the dual basis defined by $\langle\chi, X\rangle=1$. Composing ver with the evaluation map $\langle-, X\rangle: \mathfrak{h}^{\vee} \rightarrow \mathbb{C}$ defines the morphism

$$
\overline{\operatorname{ver}}^{X}: \Omega^{1}(A) \longrightarrow A, \omega \longmapsto \overline{\operatorname{ver}}^{X}(\omega):=\langle\overline{\operatorname{ver}}(\omega), X\rangle .
$$

As the right adjoint $H$-coaction on $\mathfrak{h}^{\vee}$ is trivial, it follows that

$$
\operatorname{Con}(A, \delta) \cong\left\{\omega \in \Omega^{1}(A)^{\mathrm{co} H}: \overline{\operatorname{ver}}^{X}(\omega)=\mathbf{1}\right\} .
$$

The bijection is given explicitly by $\bar{\omega}(\chi)=\omega$, for the dual basis vector $\chi \in \mathfrak{h}^{\vee}$. Analogously, the set of connections for the deformed Hopf fibration $\left(A_{\theta}, \delta\right)$ from Section 2.3 is the set of splittings of (4.16) or equivalently the set of connection forms

$$
\begin{aligned}
\operatorname{Con}\left(A_{\theta}, \delta\right): & =\left\{\bar{\omega}_{\theta} \in \operatorname{Hom}^{H}\left(\mathfrak{h}^{\vee}, \Omega^{1}\left(A_{\theta}\right)\right): \overline{\operatorname{ver}}_{\theta} \circ \bar{\omega}_{\theta}=\mathbf{1} \otimes \mathrm{id}\right\} \\
& \cong\left\{\omega_{\theta} \in \Omega^{1}\left(A_{\theta}\right)^{\operatorname{co} H}: \overline{\operatorname{ver}}_{\theta}^{X}\left(\omega_{\theta}\right)=\mathbf{1}\right\} .
\end{aligned}
$$

Notice that every connection on $(A, \delta)$ and also every connection on $\left(A_{\theta}, \delta\right)$ is strong because of (4.13) and Corollary 4.8.

Proposition 4.9. The isomorphism $\bar{\varphi}_{\theta}: \Omega^{1}\left(A_{\theta}\right) \rightarrow \Omega^{1}(A)_{\theta}$ from Proposition 4.7 defines a bijection

$$
\bar{\varphi}_{\theta}: \operatorname{Con}\left(A_{\theta}, \delta\right) \longrightarrow \operatorname{Con}(A, \delta), \quad \omega_{\theta} \longmapsto \bar{\varphi}_{\theta}\left(\omega_{\theta}\right) .
$$

Proof. Commutativity of the diagram (4.18) implies $\overline{\operatorname{ver}}^{X}\left(\bar{\varphi}_{\theta}\left(\omega_{\theta}\right)\right)=\overline{\operatorname{ver}}_{\theta}^{X}\left(\omega_{\theta}\right)=\mathbf{1}$, i.e., $\bar{\varphi}_{\theta}\left(\omega_{\theta}\right) \in \operatorname{Con}(A, \delta)$. The inverse map $\operatorname{Con}(A, \delta) \rightarrow \operatorname{Con}\left(A_{\theta}, \delta\right)$ is given by $\omega \mapsto \bar{\varphi}_{\theta}^{-1}(\omega)$.

Remark 4.10. Similar results for connections on modules were proven in $[2,3]$.

Our next aim is to refine the result of Proposition 4.9 by using more explicit features of the example under investigation. Using [25, Section 16.1], the module of Kähler 1-forms on $A$ can be computed as

$$
\Omega^{1}(A)=\frac{A \mathrm{~d} z_{1} \oplus A \mathrm{~d} z_{2} \oplus A \mathrm{~d} z_{1}^{*} \oplus A \mathrm{~d} z_{2}^{*}}{\left\langle\mathrm{~d} z_{1}^{*} z_{1}+z_{1}^{*} \mathrm{~d} z_{1}+\mathrm{d} z_{2}^{*} z_{2}+z_{2}^{*} \mathrm{~d} z_{2}\right\rangle}
$$

where the right $A$-action is defined by $s a:=a s$, for all $a \in A$ and $s \in A \mathrm{~d} z_{1} \oplus A \mathrm{~d} z_{2} \oplus A \mathrm{~d} z_{1}^{*} \oplus A \mathrm{~d} z_{2}^{*}$. The differential d: $A \rightarrow \Omega^{1}(A)$ is specified by mapping each generator $z_{1}, z_{2}, z_{1}^{*}, z_{2}^{*}$ of $A$ to the corresponding generator $\mathrm{d} z_{1}, \mathrm{~d} z_{2}, \mathrm{~d} z_{1}^{*}, \mathrm{~d} z_{2}^{*}$ of (4.21) and the Leibniz rule. Moreover, the vertical lift $\overline{\operatorname{ver}}^{X}: \Omega^{1}(A) \rightarrow A$ is given by

$$
\overline{\operatorname{ver}}^{X}\left(a \mathrm{~d} a^{\prime}\right)=a a_{\underline{0}}^{\prime} X\left(a^{\prime}{ }_{\underline{1}}\right),
$$

for all $a, a^{\prime} \in A$. 
Using braided commutativity of the deformed Hopf fibration $\left(A_{\theta}, \delta, \rho\right)$, a similar computation shows that the module of Kähler 1 -forms on $A_{\theta}$ reads as

$$
\Omega^{1}\left(A_{\theta}\right)=\frac{A_{\theta} \mathrm{d}_{\theta} z_{1} \oplus A_{\theta} \mathrm{d}_{\theta} z_{2} \oplus A_{\theta} \mathrm{d}_{\theta} z_{1}^{*} \oplus A_{\theta} \mathrm{d}_{\theta} z_{2}^{*}}{\left\langle\mathrm{~d}_{\theta} z_{1}^{*} z_{1}+z_{1}^{*} \mathrm{~d}_{\theta} z_{1}+\mathrm{d}_{\theta} z_{2}^{*} z_{2}+z_{2}^{*} \mathrm{~d}_{\theta} z_{2}\right\rangle}
$$

where the right $A_{\theta}$-action is defined using the cotriangular structure (4.15) by $s a:=R_{\theta}\left(a_{-1} \otimes\right.$ $\left.s_{-1}\right) a_{\underline{0}} s_{\underline{0}}$, for all $a \in A_{\theta}$ and $s \in A_{\theta} \mathrm{d}_{\theta} z_{1} \oplus A_{\theta} \mathrm{d}_{\theta} z_{2} \oplus A_{\theta} \mathrm{d}_{\theta} z_{1}^{*} \oplus A_{\theta} \mathrm{d}_{\theta} z_{2}^{*}$. The differential $\mathrm{d}_{\theta}: \overline{A_{\theta}} \rightarrow$ $\Omega^{1}\left(A_{\theta}\right)$ is specified by mapping each generator $z_{1}, z_{2}, z_{1}^{*}, z_{2}^{*}$ of $A_{\theta}$ to the corresponding generator $\mathrm{d}_{\theta} z_{1}, \mathrm{~d}_{\theta} z_{2}, \mathrm{~d}_{\theta} z_{1}^{*}, \mathrm{~d}_{\theta} z_{2}^{*}$ of (4.23) and the Leibniz rule. The vertical lift $\overline{\operatorname{ver}}_{\theta}^{X}: \Omega^{1}\left(A_{\theta}\right) \rightarrow A_{\theta}$ is given by

$$
\overline{\operatorname{ver}}_{\theta}^{X}\left(a \mathrm{~d}_{\theta} a^{\prime}\right)=a \star_{\theta} a_{\underline{\underline{ }}}^{\prime} X\left(a_{\underline{1}}^{\prime}\right),
$$

for all $a, a^{\prime} \in A_{\theta}$.

Lemma 4.11. The 1 -form $\omega^{0}:=z_{1}^{*} \mathrm{~d} z_{1}+z_{2}^{*} \mathrm{~d} z_{2} \in \Omega^{1}(A)$ defines a $K$-coinvariant connection on the classical Hopf fibration $(A, \delta)$. Hence, there exists a bijection

$$
\operatorname{Con}(A, \delta) \cong\left\{\omega^{0}+\alpha: \alpha \in \Omega^{1}(B)\right\} .
$$

Analogously, the 1 -form $\omega_{\theta}^{0}:=z_{1}^{*} \mathrm{~d}_{\theta} z_{1}+z_{2}^{*} \mathrm{~d}_{\theta} z_{2} \in \Omega^{1}\left(A_{\theta}\right)$ defines a $K$-coinvariant connection on the deformed Hopf fibration $\left(A_{\theta}, \delta\right)$. Hence, there exists a bijection

$$
\operatorname{Con}\left(A_{\theta}, \delta\right) \cong\left\{\omega_{\theta}^{0}+\alpha: \alpha \in \Omega^{1}(B)\right\}
$$

Proof. The one-form $\omega^{0}$ is clearly $H$-coinvariant and $K$-coinvariant. Using (4.22) and (4.19), it is found that

$$
\overline{\operatorname{ver}}^{X}\left(\omega^{0}\right)=z_{1}^{*} z_{1} X(t)+z_{2}^{*} z_{2} X(t)=z_{1}^{*} z_{1}+z_{2}^{*} z_{2}=\mathbf{1} .
$$

The bijection is a consequence of $\Omega_{\mathrm{hor}}^{1}(A)^{\mathrm{co} H}=\Omega^{1}(B)$ being the Kähler forms on $B$, cf. (4.13).

Analogously, $\omega_{\theta}^{0}$ is $H$-coinvariant and $K$-coinvariant. Using (4.24) and (4.19), it is found that

$$
\overline{\operatorname{ver}}_{\theta}^{X}\left(\omega_{\theta}^{0}\right)=z_{1}^{*} \star_{\theta} z_{1}+z_{2}^{*} \star_{\theta} z_{2}=\mathbf{1},
$$

where the last step uses (2.11). The bijection is a consequence of $\Omega_{\text {hor }}^{1}\left(A_{\theta}\right)^{\mathrm{co} H}=\Omega^{1}(B)$ being the Kähler forms on $B$, cf. Corollary 4.8 .

Proposition 4.12. Expressed in the form of Lemma 4.11, the bijection from Proposition 4.9 reads explicitly as

$$
\bar{\varphi}_{\theta}: \operatorname{Con}\left(A_{\theta}, \delta\right) \longrightarrow \operatorname{Con}(A, \delta), \quad \omega_{\theta}^{0}+\alpha \longmapsto \omega^{0}+\alpha .
$$

In other words, the affine map $\bar{\varphi}_{\theta}$ preserves the points $\omega_{\theta}^{0}$ and $\omega^{0}$, and its linear part given by the identity map id: $\Omega^{1}(B) \rightarrow \Omega^{1}(B)$.

Proof. From the explicit expression for $\bar{\varphi}_{\theta}$ given in (4.17), it is easy to confirm that $\bar{\varphi}_{\theta}\left(\omega_{\theta}^{0}\right)=\omega^{0}$ and that $\bar{\varphi}_{\theta}\left(b \mathrm{~d}_{\theta} b^{\prime}\right)=b \mathrm{~d} b^{\prime}$, for all $b, b^{\prime} \in B$, which proves our claim.

Remark 4.13. Similarly to Remark 3.4, the result in Proposition 4.12 is stronger than the isomorphisms that can be obtained by using the general theory of 2-cocycle deformations from $[10,11]$ and $[1]$. Concretely, the property that $\alpha$ is unchanged by the isomorphism $\omega_{\theta}^{0}+\alpha \mapsto \omega^{0}+\alpha$ is a particular feature of the example under investigation. 


\subsection{Gauge transformations}

We describe a notion of (infinitesimal) gauge transformations for both the deformed and the classical Hopf fibration, together with their actions on connections. We shall show that the identification of connections from Propositions 4.9 and 4.12 intertwines between the deformed and classical gauge transformations. In other words, the theory of connections and their infinitesimal gauge transformations on the deformed Hopf fibration $\left(A_{\theta}, \delta\right)$ is equivalent to that on the classical Hopf fibration $\mathbb{S}^{3} \rightarrow \mathbb{S}^{2}$.

Let us note that our notion of gauge transformations will be formalized by braided derivations and hence it makes explicitly use of the braided commutativity of $A_{\theta}$. There also exists a more flexible concept of gauge transformations given by right $H$-comodule left $B$-module automorphisms $f: A \rightarrow A$ satisfying $f(\mathbf{1})=\mathbf{1}$, see, e.g., [12]. Note that such $f$ are not required to be algebra homomorphisms. However, for commutative principal comodule algebras, this definition does not recover the usual concept of gauge transformations in classical geometry, in contrast to our more special approach by braided derivations. As a last remark, let us note that it would also be possible to describe finite gauge transformations by using the noncommutative mapping spaces from [4]. This is technically more involved and will not be discussed here.

We describe presently the case of infinitesimal gauge transformations of the deformed Hopf fibration $\left(A_{\theta}, \delta\right)$, which includes the classical case $(A, \delta)$ by setting $\theta=0$. Consider the associated left $B$-module $E_{A_{\theta}}(\mathfrak{h})=A_{\theta} \square_{H} \mathfrak{h}$, where the Lie algebra $\mathfrak{h}=\operatorname{Der}_{\epsilon}(H)$ is endowed with the adjoint left $H$-coaction, which is trivial as $U(1)$ is Abelian. Hence, $E_{A_{\theta}}(\mathfrak{h}) \cong B \otimes \mathfrak{h}$ as left $B$-modules and, using the basis element $X \in \mathfrak{h}$ from (4.19), any element $\zeta \in E_{A_{\theta}}(\mathfrak{h})$ can be written as $\zeta=b \otimes X$, for a unique $b \in B$.

Definition 4.14. An infinitesimal gauge transformation of the deformed Hopf fibration $A_{\theta}$ is an element of the left $B$-module $E_{A_{\theta}}(\mathfrak{h})$ associated to $\mathfrak{h}=\operatorname{Der}_{\epsilon}(H)$. The action of an infinitesimal gauge transformation $\zeta=b \otimes X \in E_{A_{\theta}}(\mathfrak{h})$ on $A_{\theta}$ is defined as

$$
(-)_{\theta} \triangleleft \zeta: A_{\theta} \longrightarrow A_{\theta}, \quad a \longmapsto a_{\theta} \triangleleft \zeta:=a_{\underline{0}} \star_{\theta} b X\left(a_{\underline{1}}\right) .
$$

Remark 4.15. It is easily checked that the action (4.25) of infinitesimal gauge transformations satisfies

$$
\left(a \star_{\theta} a^{\prime}\right)_{\theta} \triangleleft \zeta=a \star_{\theta}\left(a^{\prime}{ }_{\theta} \triangleleft \zeta\right)+R_{\theta}\left(\zeta_{\underline{-1}} \otimes a_{\underline{-1}}^{\prime}\right)\left(a_{\theta} \triangleleft \zeta_{\underline{0}}\right) a_{\underline{0}}^{\prime},
$$

for all $a, a^{\prime} \in A_{\theta}$ and $\zeta \in E_{A_{\theta}}(\mathfrak{h})$, i.e., $E_{A_{\theta}}(\mathfrak{h})$ acts on $A_{\theta}$ from the right by braided derivations. In particular, this action preserves the left $B$-module structure on $A_{\theta}$, i.e., $\left(b^{\prime} \star_{\theta} a\right)_{\theta} \triangleleft \zeta=b^{\prime} \star_{\theta}\left(a_{\theta} \triangleleft \zeta\right)$, for all $b^{\prime} \in B, a \in A_{\theta}$ and $\zeta \in E_{A_{\theta}}(\mathfrak{h})$.

Remark 4.16. The infinitesimal gauge transformations from Definition 4.14 are a generalization of the analogous concept in ordinary differential geometry. Given a principal $G$-bundle $P \rightarrow M$ over a manifold $M$, infinitesimal gauge transformations are given by the space of sections of the vector bundle $V P / G \rightarrow P / G \cong M$ of vertical tangent vectors modulo $G$. Using fundamental vector fields, the latter is isomorphic to the space of sections of the adjoint bundle $P \times{ }_{\text {ad }} \mathfrak{g} \rightarrow M$, which in our noncommutative example is given by $E_{A_{\theta}}(\mathfrak{h})$. The action in (4.25) is the evident generalization of the usual action of infinitesimal gauge transformations from ordinary differential geometry.

The action (4.25) can be extended to the differential graded algebra $\left(\Omega^{\bullet}\left(A_{\theta}\right), \wedge_{\theta}, \mathrm{d}_{\theta}\right)$ of deformed Kähler forms. The latter is obtained by the braided exterior algebra of the symmetric $A_{\theta}$-bimodule $\Omega^{1}\left(A_{\theta}\right)$, i.e., the wedge-product $\wedge_{\theta}$ satisfies the braided graded commutativity 
property $\lambda \wedge_{\theta} \lambda^{\prime}=(-1)^{n m} R_{\theta}\left(\lambda^{\prime}{ }_{-1} \otimes \lambda_{-1}\right) \lambda_{\underline{0}}^{\prime} \wedge_{\theta} \lambda_{\underline{0}}$, for all homogeneous $\lambda \in \Omega^{n}\left(A_{\theta}\right)$ and $\lambda^{\prime} \in \Omega^{m}\left(A_{\theta}\right)$, while the differential satisfies the graded Leibniz rule ${ }^{2}$

$$
\mathrm{d}_{\theta}\left(\lambda \wedge_{\theta} \lambda^{\prime}\right)=\lambda \wedge_{\theta}\left(\mathrm{d}_{\theta} \lambda^{\prime}\right)+(-1)^{m}\left(\mathrm{~d}_{\theta} \lambda\right) \wedge_{\theta} \lambda^{\prime},
$$

for all $\lambda \in \Omega^{\bullet}\left(A_{\theta}\right)$ and all homogeneous $\lambda^{\prime} \in \Omega^{m}\left(A_{\theta}\right)$.

Remark 4.17. Using that the isomorphism $\Omega^{1}\left(A_{\theta}\right) \cong \Omega^{1}(A)_{\theta}$ from Proposition 4.7 is an isomorphism of $(K, H)$-bicomodule $A_{\theta}$-bimodules, we obtain a canonical isomorphism of differential graded algebras

$$
\left(\Omega^{\bullet}\left(A_{\theta}\right), \wedge_{\theta}, \mathrm{d}_{\theta}\right) \cong\left(\Omega^{\bullet}(A), \wedge, \mathrm{d}\right)_{\theta},
$$

where the right hand side is the 2-cocycle deformation of the differential graded algebra of undeformed Kähler forms, see, e.g., [11, Proposition 3.17]. By a convenient abuse of notation, we shall often suppress this isomorphism in what follows, i.e., we simply identify $\lambda \wedge_{\theta} \lambda^{\prime} \in \Omega^{\bullet}\left(A_{\theta}\right)$ with the deformed wedge-product $\lambda \wedge_{\theta} \lambda^{\prime}=\sigma_{\theta}\left(\lambda_{\underline{-1}} \otimes \lambda^{\prime}{ }_{\underline{-1}}\right) \lambda_{\underline{0}} \wedge \lambda_{\underline{0}}^{\prime} \in \Omega^{\bullet}(A)_{\theta}$ and $\mathrm{d}_{\theta} \lambda \in \Omega^{\bullet}\left(A_{\theta}\right)$ with $\mathrm{d} \lambda \in \Omega^{\bullet}(A)_{\theta}$.

For every $\zeta=b \otimes X \in E_{A_{\theta}}(\mathfrak{h})$, we define the contraction map $\iota_{\zeta}^{\theta}: \Omega^{1}\left(A_{\theta}\right) \rightarrow A_{\theta}$ by setting

$$
\iota_{\zeta}^{\theta}\left(a \mathrm{~d}_{\theta} a^{\prime}\right):=a \star_{\theta}\left(a^{\prime}{ }_{\theta} \triangleleft \zeta\right)=\overline{\operatorname{ver}}_{\theta}^{X}\left(a \mathrm{~d}_{\theta} a^{\prime}\right) \star_{\theta} b,
$$

for all $a, a^{\prime} \in A_{\theta}$. Because $\overline{\operatorname{ver}}_{\theta}^{X}$ is a left $B$-module morphism, $\iota_{\zeta}^{\theta}$ preserves the left $B$-module structures too, i.e., $\iota_{\zeta}^{\theta}\left(b^{\prime} \star_{\theta} \lambda\right)=b^{\prime} \star_{\theta} \iota_{\zeta}^{\theta}(\lambda)$, for all $b^{\prime} \in B$ and $\lambda \in \Omega^{1}\left(A_{\theta}\right)$. This map can be extended to the whole of $\Omega^{\bullet}\left(A_{\theta}\right)$ as a braided anti-derivation, i.e.,

$$
\iota_{\zeta}^{\theta}\left(\lambda \wedge_{\theta} \lambda^{\prime}\right)=\lambda \wedge_{\theta} \iota_{\zeta}^{\theta}\left(\lambda^{\prime}\right)+(-1)^{m} R_{\theta}\left(\zeta_{\underline{-1}} \otimes \lambda^{\prime} \underline{-1}\right) \iota_{\zeta_{\underline{0}}}^{\theta}(\lambda) \wedge_{\theta} \lambda_{\underline{\underline{1}}}^{\prime},
$$

for all $\lambda \in \Omega^{\bullet}\left(A_{\theta}\right)$ and all homogeneous $\lambda^{\prime} \in \Omega^{m}\left(A_{\theta}\right)$.

Definition 4.18. The action of an infinitesimal gauge transformation $\zeta \in E_{A_{\theta}}(\mathfrak{h})$ on a general form $\lambda \in \Omega^{\bullet}\left(A_{\theta}\right)$ is defined by Cartan's magic formula

$$
\lambda_{\theta} \triangleleft \zeta:=\mathrm{d}_{\theta} \iota_{\zeta}^{\theta}(\lambda)+\iota_{\zeta}^{\theta}\left(\mathrm{d}_{\theta} \lambda\right) .
$$

Lemma 4.19. Let $\omega_{\theta} \in \operatorname{Con}\left(A_{\theta}, \delta\right) \subseteq \Omega^{1}\left(A_{\theta}\right)$ be a deformed connection, cf. (4.20). Then $\omega_{\theta \theta} \triangleleft \zeta=\mathrm{d}_{\theta} b$, for all $\zeta=b \otimes X \in E_{A_{\theta}}(\mathfrak{h})$.

Proof. Notice that $\iota_{\zeta}^{\theta}\left(\omega_{\theta}\right)=\overline{\operatorname{ver}}_{\theta}^{X}\left(\omega_{\theta}\right) \star_{\theta} b=1 \star_{\theta} b=b$ by (4.20). Recalling (4.23), it is found that $\omega_{\theta}=\sum_{i=1}^{4} a^{i} \mathrm{~d}_{\theta} z_{i}$, where $z_{3}=z_{1}^{*}$ and $z_{4}=z_{2}^{*}$, for some $a^{i} \in A_{\theta}$. As $\omega_{\theta}$ is $H$-coinvariant by hypothesis and $\delta\left(\mathrm{d}_{\theta} z_{i}\right)=\mathrm{d}_{\theta} z_{i} \otimes t_{i}$ with $t_{1}=t_{2}=t$ and $t_{3}=t_{4}=t^{*}$, it follows that $\delta\left(a^{i}\right)=a^{i} \otimes t_{i}^{*}$. Hence,

$$
\begin{aligned}
\omega_{\theta \theta} \triangleleft \zeta & =\mathrm{d}_{\theta} \iota_{\zeta}^{\theta}\left(\omega_{\theta}\right)+\iota_{\zeta}^{\theta}\left(\mathrm{d}_{\theta} \omega_{\theta}\right)=\mathrm{d}_{\theta} b-\sum_{i=1}^{4} \iota_{\zeta}^{\theta}\left(\mathrm{d}_{\theta} a^{i} \wedge_{\theta} \mathrm{d}_{\theta} z_{i}\right) \\
& =\mathrm{d}_{\theta} b-\sum_{i=1}^{4}\left(\mathrm{~d}_{\theta} a^{i} z_{i} X\left(t_{i}\right)-a^{i} X\left(t_{i}^{*}\right) \mathrm{d}_{\theta} z_{i}\right) b
\end{aligned}
$$

\footnotetext{
${ }^{2}$ Our variant of the graded Leibniz rule is obtained by thinking of $\mathrm{d}_{\theta}$ as acting from the right. This is a convenient choice of convention for the present work, because all other operations (e.g., covariant derivatives and gauge transformations) also act from the right.
} 


$$
\begin{aligned}
& =\mathrm{d}_{\theta} b-\sum_{i=1}^{4}\left(\mathrm{~d}_{\theta} a^{i} z_{i}+a^{i} \mathrm{~d}_{\theta} z_{i}\right) b X\left(t_{i}\right) \\
& =\mathrm{d}_{\theta} b-\mathrm{d}_{\theta}\left(\sum_{i=1}^{4} a^{i} \star_{\theta} z_{i} X\left(t_{i}\right)\right) b=\mathrm{d}_{\theta} b-\left(\mathrm{d}_{\theta} \overline{\operatorname{ver}}_{\theta}^{X}\left(\omega_{\theta}\right)\right) b=\mathrm{d}_{\theta} b,
\end{aligned}
$$

where the third line uses (4.19) and the last step follows by (4.20).

Setting $\theta=0$, we obtain the usual infinitesimal gauge transformations $E_{A}(\mathfrak{h})=A \square_{H} \mathfrak{h}$ for the classical Hopf fibration $(A, \delta)$, together with their action $\triangleleft$ on the undeformed Kähler forms $\left(\Omega^{\bullet}(A), \wedge, \mathrm{d}\right)$.

Remark 4.20. As the left $H$-coaction on $\mathfrak{h}$ is trivial, it follows that the left $B$-module isomorphism $L_{\mathfrak{h}}: E_{A}(\mathfrak{h}) \rightarrow E_{A_{\theta}}(\mathfrak{h})$ from Proposition 3.3 is the identity map, i.e., undeformed and deformed gauge transformations are identified 'on the nose'.

Proposition 4.21. The bijection $\bar{\varphi}_{\theta}: \operatorname{Con}\left(A_{\theta}, \delta\right) \rightarrow \operatorname{Con}(A, \delta)$ from Proposition 4.9 preserves infinitesimal gauge transformations, i.e.,

$$
\bar{\varphi}_{\theta}\left(\omega_{\theta \theta} \triangleleft \zeta\right)=\bar{\varphi}_{\theta}\left(\omega_{\theta}\right) \triangleleft \zeta
$$

for all $\zeta \in E_{A}(\mathfrak{h})=E_{A_{\theta}}(\mathfrak{h})$ and $\omega_{\theta} \in \operatorname{Con}\left(A_{\theta}, \delta\right)$.

Proof. Recalling the explicit expression for $\bar{\varphi}_{\theta}$ from (4.17), it is found that $\bar{\varphi}_{\theta}\left(\omega_{\theta} \triangleleft \zeta\right)=$ $\bar{\varphi}_{\theta}\left(\mathrm{d}_{\theta} b\right)=\mathrm{d} b=\bar{\varphi}_{\theta}\left(\omega_{\theta}\right) \triangleleft \zeta$.

\section{Associated gauge transformations and connections}

Let $(V, \rho) \in{ }^{H} \mathscr{M}$ be a left $H$-comodule and consider the associated left $B$-modules $E_{A}(V)=$ $A \square_{H} V$ and $E_{A_{\theta}}(V)=A_{\theta} \square_{H} V$ from Section 3. By Proposition 3.3, there exists a natural left $B$-module isomorphism $L_{V}: E_{A}(V) \rightarrow E_{A_{\theta}}(V)$. We shall show that this isomorphism does not intertwine between the actions of gauge transformations and connections on associated modules. Physically speaking, this means that the coupling of gauge and matter fields on the deformed Hopf fibration $\left(A_{\theta}, \delta\right)$ is different to that on the classical Hopf fibration $\mathbb{S}^{3} \rightarrow \mathbb{S}^{2}$.

Let us start with the associated gauge transformations. Using (4.25), there exists an induced action of $E_{A_{\theta}}(\mathfrak{h})$ on $E_{A_{\theta}}(V)$ which is given by

$$
(a \otimes v)_{\theta} \triangleleft \zeta:=\left(a_{\theta} \triangleleft \zeta\right) \otimes v=a_{\underline{0}} \star_{\theta} b \otimes v X\left(a_{\underline{1}}\right)=a \star_{\theta} b \otimes X\left(v_{\underline{-1}}\right) v_{\underline{0}},
$$

for all $a \otimes v \in E_{A_{\theta}}(V)$ and $\zeta=b \otimes X \in E_{A_{\theta}}(\mathfrak{h})$, with the last equality following from the definition of the cotensor product (3.1). Note that each $(-)_{\theta} \triangleleft \zeta: E_{A_{\theta}}(V) \rightarrow E_{A_{\theta}}(V)$ is a left $B$-module endomorphism. Hence, it defines a notion of infinitesimal gauge transformations that coincides with the analogous concepts from the more standard projective module approach to noncommutative gauge theory, see, e.g., [30]. Setting $\theta=0$, we obtain a similar expression without the $\star_{\theta}$-product, i.e.,

$$
(a \otimes v) \triangleleft \zeta=a b \otimes X\left(v_{\underline{-1}}\right) v_{\underline{0}},
$$

for all $a \otimes v \in E_{A}(V)$ and $\zeta=b \otimes X \in E_{A}(\mathfrak{h})$. Let us also recall from Remark 4.20 that $E_{A_{\theta}}(\mathfrak{h})=E_{A}(\mathfrak{h})$ are identified with the identity map $L_{\mathfrak{h}}=$ id.

Proposition 5.1. Suppose that the left $H$-coaction of $(V, \rho) \in{ }^{H} \mathscr{M}$ is non-trivial, i.e., there exists $v \in V$ such that $\rho(v) \neq \mathbf{1}_{H} \otimes v$. Then the left $B$-module isomorphism $L_{V}: E_{A}(V) \rightarrow$ $E_{A_{\theta}}(V)$ from Proposition 3.3 does not intertwine between the actions of classical (5.2) and deformed (5.1) infinitesimal gauge transformations. 
Proof. Because the $H$-coaction on $V$ is by hypothesis non-trivial, there exists a non-zero homogeneous element $a \otimes v \in A^{((m+n,-m), n)} \otimes V^{n}$ with $n \neq 0$. Consider any infinitesimal gauge transformation $\zeta=b \otimes X \in E_{A_{\theta}}(\mathfrak{h})=E_{A}(\mathfrak{h})$ with $b \in A^{\left(\left(m^{\prime},-m^{\prime}\right), 0\right)}$ a non-zero homogeneous element with $m^{\prime} \neq 0$. Using (3.6) and (5.2), we compute

$$
L_{V}((a \otimes v) \triangleleft \zeta)=\mathrm{e}^{\pi \mathrm{i} \theta\left(m+m^{\prime}\right) n} a b \otimes X\left(v_{\underline{-1}}\right) v_{\underline{\underline{0}}} .
$$

Using also (5.1) and (3.5), we compute

$$
L_{V}(a \otimes v)_{\theta} \triangleleft \zeta=\mathrm{e}^{\pi \mathrm{i} \theta\left(m-m^{\prime}\right) n} a b \otimes X\left(v_{\underline{-1}}\right) v_{\underline{0}} .
$$

The phase factors differ because $n \neq 0$ and $m^{\prime} \neq 0$, which proves our claim.

Remark 5.2. The result of Proposition 5.1 that $L_{V}$ does not intertwine between classical and deformed gauge transformations can also be understood from the following more heuristic argument: Gauge transformations of left B-modules act from the right (cf. (5.1) where $b$ is to the right of $a$ ) and hence they are in general not intertwined by the left $B$-module isomorphism $L_{V}$.

Let us now discuss the covariant derivatives (4.4) and their corresponding associated connections (4.5). We fix any connection form $\omega=\omega^{0}+\alpha \in \operatorname{Con}(A, \delta)$ on the classical Hopf fibration (cf. Lemma 4.11) and determine the corresponding connection form on the deformed Hopf fibration $\left(A_{\theta}, \delta\right)$ via the bijection given in Proposition 4.12, i.e., $\omega_{\theta}=\omega_{\theta}^{0}+\alpha \in \operatorname{Con}\left(A_{\theta}, \delta\right)$ with the same $\alpha$. For later convenience, we consider the canonical lift of the covariant derivative $\bar{D}: A \rightarrow \Omega_{\mathrm{hor}}^{1}(A)$ to the graded subalgebra $\left(\Omega_{\mathrm{hor}}^{\bullet}(A), \wedge\right) \subseteq\left(\Omega^{\bullet}(A), \wedge\right)$ of horizontal Kähler forms. Note that $\Omega_{\text {hor }}^{\bullet}(A)=\Omega^{\bullet}(B) A$ because of (4.13) and graded commutativity of $\wedge$. This yields a linear map $\bar{D}: \Omega_{\mathrm{hor}}^{\bullet}(A) \rightarrow \Omega_{\mathrm{hor}}^{\bullet+1}(A)$, which explicitly reads as

$$
\bar{D}(\lambda)=\mathrm{d} \lambda-\lambda_{\underline{0}} \wedge \omega X\left(\lambda_{\underline{1}}\right)=\mathrm{d} \lambda-\lambda_{\underline{0}} \wedge\left(\omega^{0}+\alpha\right) X\left(\lambda_{\underline{1}}\right),
$$

for all $\lambda \in \Omega_{\text {hor }}^{\bullet}(A)$. By construction, $\bar{D}$ satisfies the graded Leibniz rule

$$
\bar{D}(\beta \wedge \lambda)=(-1)^{m} \mathrm{~d} \beta \wedge \lambda+\beta \wedge \bar{D}(\lambda),
$$

for all $\beta \in \Omega^{\bullet}(B)$ and all homogeneous $\lambda \in \Omega_{\text {hor }}^{m}(A)$. (Compare this with the similar graded Leibniz rule for $\mathrm{d}$ in (4.26).) The canonical lift $\bar{D}_{\theta}: \Omega_{\mathrm{hor}}^{\bullet}\left(A_{\theta}\right) \rightarrow \Omega_{\mathrm{hor}}^{\bullet+1}\left(A_{\theta}\right)$ of the corresponding deformed covariant derivative to the graded subalgebra $\left(\Omega_{\text {hor }}^{\bullet}\left(A_{\theta}\right), \wedge_{\theta}\right) \subseteq\left(\Omega^{\bullet}\left(A_{\theta}\right), \wedge_{\theta}\right)$ of horizontal deformed Kähler forms explicitly reads as

$$
\bar{D}_{\theta}(\lambda)=\mathrm{d}_{\theta} \lambda-\lambda_{\underline{0}} \wedge_{\theta} \omega_{\theta} X\left(\lambda_{\underline{1}}\right)=\mathrm{d}_{\theta} \lambda-\lambda_{\underline{0}} \wedge_{\theta}\left(\omega_{\theta}^{0}+\alpha\right) X\left(\lambda_{\underline{1}}\right),
$$

for all $\lambda \in \Omega_{\text {hor }}^{\bullet}\left(A_{\theta}\right)$. By construction, $\bar{D}_{\theta}$ satisfies the graded Leibniz rule

$$
\bar{D}_{\theta}\left(\beta \wedge_{\theta} \lambda\right)=(-1)^{m} \mathrm{~d}_{\theta} \beta \wedge_{\theta} \lambda+\beta \wedge_{\theta} \bar{D}_{\theta}(\lambda),
$$

for all $\beta \in \Omega^{\bullet}(B)$ and all homogeneous $\lambda \in \Omega_{\text {hor }}^{m}\left(A_{\theta}\right)$. Notice that $\Omega_{\text {hor }}^{\bullet}\left(A_{\theta}\right)=\Omega^{\bullet}(B) A_{\theta}$ because of Corollary 4.8 and braided graded commutativity of $\wedge_{\theta}$.

Regarding both $\Omega_{\text {hor }}^{\bullet}(A)=\Omega^{\bullet}(B) A$ and $\Omega_{\text {hor }}^{\bullet}\left(A_{\theta}\right)=\Omega^{\bullet}(B) A_{\theta}$ as graded left $\Omega^{\bullet}(B)$-modules, we obtain in analogy to Proposition 3.3 a graded left $\Omega^{\bullet}(B)$-module isomorphism $L^{\bullet}: \Omega_{\text {hor }}^{\bullet}(A) \rightarrow$ $\Omega_{\text {hor }}^{\bullet}\left(A_{\theta}\right)$ by setting

$$
L^{\bullet}(\lambda):=\mathrm{e}^{\pi \mathrm{i} \theta m n} \lambda
$$

for all homogeneous elements $\lambda \in \Omega_{\text {hor }}^{\bullet}(A)^{((m+n,-m), n)} \subseteq \Omega_{\text {hor }}^{\bullet}(A)$. 
Proposition 5.3. Suppose that the chosen connection form $\omega=\omega^{0}+\alpha \in \operatorname{Con}(A, \delta)$ is not $K$ coinvariant. Then the left $\Omega^{\bullet}(B)$-module isomorphism $L^{\bullet}: \Omega_{\mathrm{hor}}^{\bullet}(A) \rightarrow \Omega_{\mathrm{hor}}^{\bullet}\left(A_{\theta}\right)$ given in (5.6) does not intertwine between the classical (5.4) and the deformed (5.5) covariant derivatives.

Proof. Let us decompose $\alpha$ as $\alpha=\sum_{m^{\prime} \in \mathbb{Z}} \alpha_{m^{\prime}}$, where $\alpha_{m^{\prime}} \in \Omega^{1}(B)^{\left(\left(m^{\prime},-m^{\prime}\right), 0\right)}$, and note that by hypothesis there exists $m^{\prime} \neq 0$ such that $\alpha_{m^{\prime}} \neq 0$. For any homogeneous $\lambda \in \Omega_{\text {hor }}^{\bullet}(A)^{((m+n,-m), n)}$ with $n \neq 0$, we find after a short calculation using the identification explained in Remark 4.17 and the analogue of (3.5) for deformed wedge-products

$$
\left(L^{\bullet}\right)^{-1}\left(\bar{D}_{\theta} L^{\bullet}(\lambda)\right)=\mathrm{d} \lambda-\lambda_{\underline{0}} \wedge\left(\omega^{0}+\sum_{m^{\prime} \in \mathbb{Z}} \mathrm{e}^{-2 \pi \mathrm{i} \theta m^{\prime} n} \alpha_{m^{\prime}}\right) X\left(\lambda_{\underline{1}}\right),
$$

which is different to $\bar{D}(\lambda)$ given in (5.4) because $\alpha_{m^{\prime}} \neq 0$ for some $m^{\prime} \neq 0$.

Remark 5.4. Similarly to Remark 5.2 , this feature can also be understood from the fact that covariant derivatives act from the right on the left $\Omega^{\bullet}(B)$-modules $\Omega_{\mathrm{hor}}^{\bullet}(A)$ and $\Omega_{\text {hor }}^{\bullet}\left(A_{\theta}\right)$ and hence they are in general not intertwined by the left $\Omega^{\bullet}(B)$-module isomorphism $L^{\bullet}$. An exception is the case of a $K$-coinvariant connection form $\omega=\omega^{0}+\alpha \in \operatorname{Con}(A, \delta)$, i.e., $\alpha \in \Omega^{1}(B)^{((0,0), 0)}$.

Remark 5.5. As a side-remark, we note the following expressions for the classical and deformed curvatures, which are defined as the squares of the corresponding covariant derivatives. In the classical case, we obtain by a direct calculation using (5.4)

$$
\begin{aligned}
\bar{D}^{2}(\lambda) & =\mathrm{d}\left(\mathrm{d} \lambda-\lambda_{\underline{0}} \wedge \omega X\left(\lambda_{\underline{1}}\right)\right)-\left(\mathrm{d} \lambda_{\underline{0}}-\lambda_{\underline{0}_{\underline{0}}} \wedge \omega X\left(\lambda_{\underline{0}_{1}}\right)\right) \wedge \omega X\left(\lambda_{\underline{1}}\right) \\
& =-\lambda_{\underline{0}} \wedge \mathrm{d} \omega X\left(\lambda_{\underline{1}}\right)+\lambda_{\underline{0}} \wedge \omega \wedge \omega X\left(\lambda_{\underline{1}_{1}}\right) X\left(\lambda_{\underline{1}_{\underline{2}}}\right) \\
& =-\lambda_{\underline{0}} \wedge \mathrm{d} \omega X\left(\lambda_{\underline{1}}\right),
\end{aligned}
$$

where in the last equality we used that by graded commutativity $\omega \wedge \omega=-\omega \wedge \omega=0$. In the deformed case, we obtain by a similar direct calculation using (5.5)

$$
\bar{D}_{\theta}^{2}(\lambda)=-\lambda_{\underline{0}} \wedge_{\theta} \mathrm{d}_{\theta} \omega_{\theta} X\left(\lambda_{\underline{1}}\right) .
$$

Here we also use that $\omega_{\theta}=\omega_{\theta}^{0}+\alpha$, where $\omega_{\theta}^{0}$ is $K$-coinvariant and $\alpha \in \Omega^{1}(B)$, hence by braided graded commutativity $\omega_{\theta} \wedge_{\theta} \omega_{\theta}=-R_{\theta}\left(\left(\omega_{\theta}\right)_{\underline{-1}} \otimes\left(\omega_{\theta}\right)_{\underline{-1}}\right)\left(\omega_{\theta}\right)_{\underline{0}} \wedge_{\theta}\left(\omega_{\theta}\right)_{\underline{0}}=-\omega_{\theta} \wedge_{\theta} \omega_{\theta}=0$. Under the identification explained in Remark 4.17, the underlying deformed curvature form $\mathrm{d}_{\theta} \omega_{\theta}$ coincides with the classical curvature form $\mathrm{d} \omega$. However, by a similar argument as above, the actions of these curvature forms on, respectively, $\Omega_{\text {hor }}^{\bullet}\left(A_{\theta}\right)$ and $\Omega_{\text {hor }}^{\bullet}(A)$ are in general not intertwined by the isomorphism $L^{\bullet}$.

Let now $(V, \rho) \in{ }^{H} \mathscr{M}$ be any left $H$-comodule and consider the corresponding associated connections $\bar{\nabla}: E_{A}(V) \rightarrow \Omega^{1}(B) \otimes_{B} E_{A}(V)$ and $\bar{\nabla}_{\theta}: E_{A_{\theta}}(V) \rightarrow \Omega^{1}(B) \otimes_{B} E_{A_{\theta}}(V)$. The latter are obtained concretely by composing, respectively, $\bar{D} \otimes \mathrm{id}: A \square_{H} V \rightarrow \Omega_{\mathrm{hor}}^{1}(A) \square_{H} V$ and $\bar{D}_{\theta} \otimes$ id: $A_{\theta} \square_{H} V \rightarrow \Omega_{\text {hor }}^{1}\left(A_{\theta}\right) \square_{H} V$ with the inverses of the corresponding left $B$-module isomorphisms

$$
\begin{aligned}
& \Omega^{1}(B) \otimes_{B} E_{A}(V) \longrightarrow \Omega_{\text {hor }}^{1}(A) \square_{H} V, \beta \otimes_{B}(a \otimes v) \longmapsto \beta a \otimes v, \\
& \Omega^{1}(B) \otimes_{B} E_{A_{\theta}}(V) \longrightarrow \Omega_{\text {hor }}^{1}\left(A_{\theta}\right) \square_{H} V, \beta \otimes_{B}(a \otimes v) \longmapsto \beta \star_{\theta} a \otimes v .
\end{aligned}
$$

These isomorphisms are compatible with the isomorphisms $L_{V}$ in Proposition 3.3 and $L^{\bullet}$ in (5.6) in the sense that the diagram

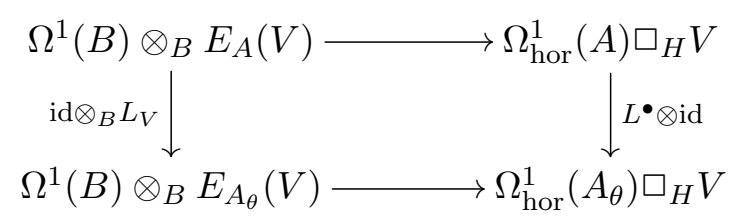

commutes. The following result is then an immediate consequence of Proposition 5.3. 
Corollary 5.6. Suppose that the left $H$-coaction of $(V, \rho) \in{ }^{H} \mathscr{M}$ is non-trivial and that the connection form $\omega \in \operatorname{Con}(A, \delta)$ is not $K$-coinvariant. Then the left B-module isomorphism $L_{V}: E_{A}(V) \rightarrow E_{A_{\theta}}(V)$ from Proposition 3.3 does not intertwine between the classical and the deformed associated connections, i.e., $\bar{\nabla}_{\theta} \circ L_{V} \neq\left(\mathrm{id} \otimes_{B} L_{V}\right) \circ \bar{\nabla}$.

Example 5.7. Let us consider the explicit example where $(V, \rho)=\mathbb{C}^{(n)}:=\left(\mathbb{C}, \rho^{(n)}\right)$ is the 1-dimensional irreducible left $H$-comodule given by $\rho^{(n)}: c \mapsto t^{n} \otimes c$, for some $n \in \mathbb{Z}$. The associated left $B$-modules $E_{A}\left(\mathbb{C}^{(n)}\right)=\bigoplus_{m \in \mathbb{Z}} A^{((m+n,-m), n)}$ and $E_{A_{\theta}}\left(\mathbb{C}^{(n)}\right)=\bigoplus_{m \in \mathbb{Z}} A^{((m+n,-m), n)}$ describe a matter field of charge $n \in \mathbb{Z}$ on the classical and deformed Hopf fibrations respectively. Every deformed gauge transformation by $\zeta \in E_{A_{\theta}}(\mathfrak{h})$ can be interpreted in terms of an undeformed gauge transformation by defining an effective gauge transformation parameter $\zeta_{\theta}^{(n)} \in E_{A}(\mathfrak{h})$ according to

$$
\lambda \triangleleft \zeta_{\theta}^{(n)}:=L_{V}^{-1}\left(L_{V}(\lambda)_{\theta} \triangleleft \zeta\right)
$$

for all $\lambda \in E_{A}\left(\mathbb{C}^{(n)}\right)$. To compute $\zeta_{\theta}^{(n)}$ explicitly, we decompose $\zeta$ as $\zeta=\sum_{m^{\prime} \in \mathbb{Z}} \zeta_{m^{\prime}}$, where $\zeta_{m^{\prime}} \in A^{\left(\left(m^{\prime},-m^{\prime}\right), 0\right)}$, and obtain from (5.3) the expression

$$
\zeta_{\theta}^{(n)}=\sum_{m^{\prime} \in \mathbb{Z}} \mathrm{e}^{-2 \pi \mathrm{i} \theta m^{\prime} n} \zeta_{m^{\prime}}
$$

where we also used Remark 4.20 to identify $E_{A_{\theta}}(\mathfrak{h})=E_{A}(\mathfrak{h})$ with the identity map $L_{\mathfrak{h}}=$ id.

Analogously, the deformed covariant derivative can be interpreted in terms of an undeformed covariant derivative by defining

$$
\bar{D}_{\theta}^{(n)}(\lambda):=\left(L^{\bullet}\right)^{-1}\left(\bar{D}_{\theta} L^{\bullet}(\lambda)\right)
$$

for all $\lambda \in E_{A}\left(\mathbb{C}^{(n)}\right)$. Decomposing the 1-form $\alpha \in \Omega^{1}(B)$ in (5.5) as $\alpha=\sum_{m^{\prime} \in \mathbb{Z}} \alpha_{m^{\prime}}$, where $\alpha_{m^{\prime}} \in \Omega^{1}(B)^{\left(\left(m^{\prime},-m^{\prime}\right), 0\right)}$, and using (5.7), we obtain

$$
\bar{D}_{\theta}^{(n)}(\lambda)=\mathrm{d} \lambda-\lambda_{\underline{0}} \wedge\left(\omega^{0}+\alpha_{\theta}^{(n)}\right) X\left(\lambda_{\underline{1}}\right),
$$

where the effective gauge potential is given by

$$
\alpha_{\theta}^{(n)}=\sum_{m^{\prime} \in \mathbb{Z}} \mathrm{e}^{-2 \pi \mathrm{i} \theta m^{\prime} n} \alpha_{m^{\prime}}
$$

Summing up, the physical effect of the studied deformation on charged matter fields is that they experience effective gauge transformations and effective covariant derivatives that depend on both the charge $n \in \mathbb{Z}$ and on the deformation parameter $\theta \in \mathbb{R}$.

\section{Outlook: Towards an explanation via homotopy equivalence}

In this section, we construct a suitable type of homotopy equivalence between the classical and deformed Hopf fibrations. The concept of homotopy equivalence presented here is slightly different from the one proposed by Kassel and Schneider [29] in the sense that for our purposes it is more convenient to work with a larger algebra of complex-valued functions on the real line $\mathbb{R}$ than the one given by the polynomial algebra $\mathbb{C}[y]$ in one generator $y$. In particular, we would like the exponential functions $y \mapsto \mathrm{e}^{\mathrm{i} c y}$, for $c \in \mathbb{R}$, to be contained in this algebra in order to obtain a homotopy equivalence that relates the exponentiated deformation parameter $q=\mathrm{e}^{2 \pi \mathrm{i} \theta}$ to the classical value 1 . 
Let us fix any $*$-subalgebra $\overline{\mathcal{O}}(\mathbb{R}) \subseteq \operatorname{Fun}(\mathbb{R}, \mathbb{C})$ of the $*$-algebra of complex-valued functions on the real line that contains the monomials $y^{n} \in \overline{\mathcal{O}}(\mathbb{R})$, for $n \geq 0$, and also the exponential functions $\mathrm{e}^{\mathrm{i} c y} \in \overline{\mathcal{O}}(\mathbb{R})$, for $c \in \mathbb{R}$. For example, we could choose the algebra of analytic functions $C^{\omega}(\mathbb{R}, \mathbb{C})$, the algebra of smooth functions $C^{\infty}(\mathbb{R}, \mathbb{C})$ or the algebra of continuous functions $C^{0}(\mathbb{R}, \mathbb{C})$. Note that the polynomial algebra $\mathbb{C}[y]$ used in [29] does not satisfy our criteria because it does not contain the exponential functions. Consider the two evaluation maps ev $e_{p}: \overline{\mathcal{O}}(\mathbb{R}) \rightarrow \mathbb{C}, f \mapsto f(p)$, for $p=0,1$, and note that they are algebra homomorphisms. The following definition generalizes [29, Definition 2.3] to the new concept of $\overline{\mathcal{O}}(\mathbb{R})$-homotopy equivalence.

Definition 6.1. Let $H$ be a Hopf algebra and $B$ an algebra. Let $A_{0}$ and $A_{1}$ be two principal $H$-comodule algebras over $B$. We write $A_{0} \sim A_{1}$ if there exists a principal $H \otimes \overline{\mathcal{O}}(\mathbb{R})$-comodule algebra $A$ over $B \otimes \overline{\mathcal{O}}(\mathbb{R})$ with ground ring $\overline{\mathcal{O}}(\mathbb{R})$ such that $\operatorname{ev}_{p_{*}}(A):=A \otimes_{\overline{\mathcal{O}}(\mathbb{R})} \mathbb{C} \cong A_{p}$ are isomorphic as $H$-comodule algebras, for $p=0,1 .^{3}$

$\overline{\mathcal{O}}(\mathbb{R})$-homotopy equivalence of principal $H$-comodule algebras over $B$ is the equivalence relation $\approx$ generated by $\sim$.

In the following, we let $H=\mathcal{O}(U(1))$ and $B=\mathcal{O}\left(\mathbb{S}^{2}\right)$ as in Section 2.2. We denote the classical Hopf fibration from Section 2.2 by $A_{0}$ and the deformed Hopf fibration, with a fixed deformation parameter $\theta \in \mathbb{R}$, from Section 2.3 by $A_{1}$. Our aim is to construct for this scenario a principal $H \otimes \overline{\mathcal{O}}(\mathbb{R})$-comodule algebra $A$ over $B \otimes \overline{\mathcal{O}}(\mathbb{R})$ with ground ring $\overline{\mathcal{O}}(\mathbb{R})$, as in Definition 6.1 , that implements an $\overline{\mathcal{O}}(\mathbb{R})$-homotopy equivalence between the classical Hopf fibration $A_{0}$ and the deformed Hopf fibration $A_{1}$. This requires a few preparations. Let us first note that the change of base ring functor $(-) \otimes \overline{\mathcal{O}}(\mathbb{R}): \mathscr{M}_{\mathbb{C}} \rightarrow \mathscr{M}_{\overline{\mathcal{O}}(\mathbb{R})}$ maps free algebras over $\mathbb{C}$ to the corresponding free algebras over $\overline{\mathcal{O}}(\mathbb{R})$ because it is monoidal, i.e., $(V \otimes \overline{\mathcal{O}}(\mathbb{R})) \otimes_{\overline{\mathcal{O}}(\mathbb{R})}(W \otimes \overline{\mathcal{O}}(\mathbb{R})) \cong(V \otimes W) \otimes \overline{\mathcal{O}}(\mathbb{R})$ for all $V, W \in \mathscr{M}_{\mathbb{C}}$, and it preserves all small colimits. Because of the latter property, it also follows that $(-) \otimes \overline{\mathcal{O}}(\mathbb{R})$ maps finitely presented algebras over $\mathbb{C}$ to the corresponding finitely presented algebras over $\overline{\mathcal{O}}(\mathbb{R})$. This implies that $H \otimes \overline{\mathcal{O}}(\mathbb{R})$ is the commutative $*$-algebra over $\overline{\mathcal{O}}(\mathbb{R})$ generated by one generator $t$ that satisfies the circle relation $t^{*} t=\mathbf{1}$, and that $B \otimes \overline{\mathcal{O}}(\mathbb{R})$ is the commutative $*$-algebra over $\overline{\mathcal{O}}(\mathbb{R})$ generated by two generators $z$ and $x$ that satisfy $x^{*}=x$ and the 2 -sphere relation $z^{*} z+x^{2}=\mathbf{1}$. In other words, $H \otimes \overline{\mathcal{O}}(\mathbb{R})$ and $B \otimes \overline{\mathcal{O}}(\mathbb{R})$ are described by the same formulae as the ones for $H$ and $B$ in Section 2.2 , however the base ring is now $\overline{\mathcal{O}}(\mathbb{R})$ instead of $\mathbb{C}$.

Let us denote by $Q \in \overline{\mathcal{O}}(\mathbb{R})$ the function given by

$$
Q: \mathbb{R} \longrightarrow \mathbb{C}, \quad y \longmapsto Q(y)=\mathrm{e}^{2 \pi \mathrm{i} \theta y} .
$$

Note that $Q(0)=1$ and $Q(1)=q=\mathrm{e}^{2 \pi \mathrm{i} \theta}$. We define $A$ to be the noncommutative $*$-algebra over $\overline{\mathcal{O}}(\mathbb{R})$ generated by two generators $z_{1}$ and $z_{2}$ satisfying the commutation relations

$$
z_{1} z_{1}^{*}=z_{1}^{*} z_{1}, \quad z_{2} z_{2}^{*}=z_{2}^{*} z_{2}, \quad z_{1} z_{2}=Q z_{2} z_{1}, \quad z_{1} z_{2}^{*}=Q^{-1} z_{2}^{*} z_{1},
$$

and the 3 -sphere relation

$$
z_{1}^{*} z_{1}+z_{2}^{*} z_{2}=\mathbf{1}
$$

Compare this to (2.10) and (2.11). We further endow $A$ with a right $H \otimes \overline{\mathcal{O}}(\mathbb{R})$-comodule structure $\delta: A \rightarrow A \otimes_{\overline{\mathcal{O}}(\mathbb{R})}(H \otimes \overline{\mathcal{O}}(\mathbb{R}))$ by using the same formulae (2.3) for the generators as for the case where the base ring is $\mathbb{C}$.

\footnotetext{
${ }^{3}$ Here $\operatorname{ev}_{p_{*}}$ denotes the usual change of base ring construction along the algebra maps ev $: \overline{\mathcal{O}}(\mathbb{R}) \rightarrow \mathbb{C}$. It is given concretely by the relative tensor product $\operatorname{ev}_{p_{*}}(A)=A \otimes_{\overline{\mathcal{O}}(\mathbb{R})} \mathbb{C}$ where the left $\overline{\mathcal{O}}(\mathbb{R})$-module structure on $\mathbb{C}$ is defined by $\overline{\mathcal{O}}(\mathbb{R}) \otimes \mathbb{C} \stackrel{\text { evp } \stackrel{\text { id }}{\longrightarrow}}{\longrightarrow} \otimes \mathbb{C} \stackrel{\mu_{\mathbb{C}}}{\longrightarrow} \mathbb{C}$.
} 
Lemma 6.2. The $H \otimes \overline{\mathcal{O}}(\mathbb{R})$-comodule algebra $(A, \delta)$ described above is a principal comodule algebra with subalgebra of coinvariants isomorphic to $B \otimes \overline{\mathcal{O}}(\mathbb{R})$.

Proof. The proof is analogous to the one for algebras over $\mathbb{C}$, cf. Sections 2.2 and 2.3 and also [19].

We can now show that this principal comodule algebra implements an $\overline{\mathcal{O}}(\mathbb{R})$-homotopy equivalence between the classical Hopf fibration $A_{0}$ and the deformed Hopf fibration $A_{1}$.

Proposition 6.3. There exist isomorphisms of $H$-comodule algebras $\operatorname{ev}_{p_{*}}(A) \cong A_{p}$, for $p=$ 0,1. Hence, the classical Hopf fibration $A_{0}$ is $\overline{\mathcal{O}}(\mathbb{R})$-homotopy equivalent to the deformed Hopf fibration $A_{1}$ for any value of the deformation parameter $\theta \in \mathbb{R}$.

Proof. Using the map $\mathbb{C} \rightarrow \overline{\mathcal{O}}(\mathbb{R}), c \mapsto c$ that assigns to a complex number the corresponding constant function on $\mathbb{R}$, we can consider $A$ as an algebra over $\mathbb{C}$. The homomorphism of $\mathbb{C}$ algebras $A \rightarrow \operatorname{ev}_{p_{*}}(A)=A \otimes_{\overline{\mathcal{O}}(\mathbb{R})} \mathbb{C}, a \mapsto a \otimes_{\overline{\mathcal{O}}(\mathbb{R})} 1$ is surjective as $a \otimes_{\overline{\mathcal{O}}(\mathbb{R})} c=a c \otimes_{\overline{\mathcal{O}}(\mathbb{R})} 1$. The kernel of this map is the two-sided ideal of the $\mathbb{C}$-algebra $A$ generated by $(f-f(p)) \mathbf{1} \in A$, for all $f \in \overline{\mathcal{O}}(\mathbb{R})$. Hence, $\operatorname{ev}_{p_{*}}(A)$ is isomorphic to the quotient $\mathbb{C}$-algebra $A /((f-f(p)) \mathbf{1}: f \in \overline{\mathcal{O}}(\mathbb{R}))$, i.e., the evaluation of all coefficient functions in $\overline{\mathcal{O}}(\mathbb{R})$ at $p \in \mathbb{R}$. Recalling (6.1), (6.2) and (6.3), we obtain that $\operatorname{ev}_{0 *}(A)$ is isomorphic to the classical 3-sphere due to $Q(0)=1$ and that $e_{1 *}(A)$ is isomorphic to the deformed 3 -sphere with deformation parameter $\theta$ as $Q(1)=q=\mathrm{e}^{2 \pi \mathrm{i} \theta}$. These isomorphisms are clearly compatible with the $H$-coactions.

We finish this section by explaining why we believe that Proposition 6.3 is the conceptual reason for the results in Section 3.2 stating that the associated module functors for the classical and deformed Hopf fibration are naturally isomorphic. The following argument is inspired by [29, Remark 2.4(4)]. Let us first note that the underlying vector space of the Hopf algebra $H=\mathcal{O}(U(1))$ admits a decomposition $H=\bigoplus_{n \in \mathbb{Z}} C_{n}$, where $C_{n}=\mathbb{C} t^{n}$ is the (1-dimensional) vector space spanned by the $n$-th power of the generator $t$. (Recall that $t^{-1}=t^{*}$.) As $\Delta\left(t^{n}\right)=$ $t^{n} \otimes t^{n}$, each $C_{n}$ is a left $H$-comodule via the coaction $\Delta: C_{n} \rightarrow H \otimes C_{n}$. It follows that $A \cong$ $\bigoplus_{n \in \mathbb{Z}} A \square_{H} C_{n}$ is a direct sum of associated modules $E_{A}\left(C_{n}\right)=A \square_{H} C_{n}$ with $C_{n} \in{ }^{H} \mathscr{M}_{\text {fin }}$ finitedimensional. By [13], each $E_{A}\left(C_{n}\right)$ is a finitely generated projective left $B \otimes \overline{\mathcal{O}}(\mathbb{R})$-module and hence it defines an element $\left[E_{A}\left(C_{n}\right)\right]$ of the zeroth $K$-theory group $K_{0}(B \otimes \overline{\mathcal{O}}(\mathbb{R}))$. Analogously, there exists a decomposition $A_{p} \cong \bigoplus_{n \in \mathbb{Z}} A_{p} \square_{H} C_{n}$ into a direct sum of associated modules $E_{A_{p}}\left(C_{n}\right)=A_{p} \square_{H} C_{n}$ and we obtain elements $\left[E_{A_{p}}\left(C_{n}\right)\right] \in K_{0}(B)$, for $p=0,1$. Because of Proposition 6.3 , we know that $\left[E_{A_{p}}\left(C_{n}\right)\right] \in K_{0}(B)$ is the image under

$$
K_{0}\left(\mathrm{ev}_{p_{*}}\right): K_{0}(B \otimes \overline{\mathcal{O}}(\mathbb{R})) \longrightarrow K_{0}(B)
$$

of the element $\left[E_{A}\left(C_{n}\right)\right] \in K_{0}(B \otimes \overline{\mathcal{O}}(\mathbb{R}))$, for $p=0,1$. The same holds true for the modules $E_{A}(V), E_{A_{0}}(V)$ and $E_{A_{1}}(V)$ associated to any finite-dimensional left $H$-comodule as any such $V$ decomposes as a finite direct sum of $C_{n}$ 's.

If we could prove that the $K_{0}$-groups are invariant under $\overline{\mathcal{O}}(\mathbb{R})$-homotopy equivalences, the concrete result of Section 3.2, namely that the associated modules $E_{A_{0}}(V)$ and $E_{A_{1}}(V)$ of the classical and deformed Hopf fibrations are isomorphic, would follow from the more conceptual argument in this section. While results in this direction are available for homotopy equivalences described by the polynomial algebra $\mathbb{C}[y]$, see, e.g., [5], we are not aware of generalizations to the setup of $\overline{\mathcal{O}}(\mathbb{R})$-homotopy equivalences. We expect that addressing this question might provide some insights on appropriate choices of the algebra $\overline{\mathcal{O}}(\mathbb{R})$, which we have left unspecified above, besides assuming that it contains exponential functions and polynomials. Developing a theory of $\overline{\mathcal{O}}(\mathbb{R})$-homotopy equivalences would be useful and interesting also for other typical examples in noncommutative geometry where the deformation parameter appears in an exponentiated form $q=\mathrm{e}^{2 \pi \mathrm{i} \theta}$. We hope to come back to this issue in a future work. 


\section{Acknowledgments}

We would like to thank Christian Lomp and Alexander Vishik for useful comments related to this work. We also would like to thank the anonymous referees for their valuable comments and suggestions that helped us to improve this manuscript. The research of T.B. was partially supported by the Polish National Science Centre grant 2016/21/B/ST1/02438. A.S. gratefully acknowledges the financial support of the Royal Society (UK) through a Royal Society University Research Fellowship, a Research Grant and an Enhancement Award.

\section{References}

[1] Aschieri P., Bieliavsky P., Pagani C., Schenkel A., Noncommutative principal bundles through twist deformation, Comm. Math. Phys. 352 (2017), 287-344, arXiv:1604.03542.

[2] Aschieri P., Schenkel A., Noncommutative connections on bimodules and Drinfeld twist deformation, Adv. Theor. Math. Phys. 18 (2014), 513-612, arXiv:1210.0241.

[3] Barnes G.E., Schenkel A., Szabo R.J., Nonassociative geometry in quasi-Hopf representation categories II: Connections and curvature, J. Geom. Phys. 106 (2016), 234-255, arXiv:1507.02792.

[4] Barnes G.E., Schenkel A., Szabo R.J., Mapping spaces and automorphism groups of toric noncommutative spaces, Lett. Math. Phys. 107 (2017), 1591-1628, arXiv:1606.04775.

[5] Bass H., Algebraic K-theory, W. A. Benjamin, Inc., New York - Amsterdam, 1968.

[6] Baum P.F., Hajac P.M., Matthes R., Szymański W., Noncommutative geometry approach to principal and associated bundles, arXiv:math.DG/0701033.

[7] Beggs E.J., Brzeziński T., An explicit formula for a strong connection, Appl. Categ. Structures 16 (2008), 57-63, arXiv:math.QA/0606586.

[8] Beggs E.J., Majid S., Quantum Riemannian geometry, Grundlehren der mathematischen Wissenschaften, Vol. 355, Springer, Cham, 2020.

[9] Böhm G., Brzeziński T., Strong connections and the relative Chern-Galois character for corings, Int. Math. Res. Not. 2005 (2005), 2579-2625, arXiv:math.RA/0503469.

[10] Brain S., Landi G., Moduli spaces of non-commutative instantons: gauging away non-commutative parameters, Q. J. Math. 63 (2012), 41-86, arXiv:0909.4402.

[11] Brain S., Landi G., van Suijlekom W.D., Moduli spaces of instantons on toric noncommutative manifolds, Adv. Theor. Math. Phys. 17 (2013), 1129-1193, arXiv:1204.2148.

[12] Brzeziński T., Translation map in quantum principal bundles, J. Geom. Phys. 20 (1996), 349-370, arXiv:hepth/9407145.

[13] Brzeziński T., Hajac P.M., The Chern-Galois character, C. R. Math. Acad. Sci. Paris 338 (2004), 113-116, arXiv:math.KT/0306436.

[14] Brzeziński T., Hajac P.M., Galois-type extensions and equivariant projectivity, arXiv:0901.0141.

[15] Brzeziński T., Janelidze G., Maszczyk T., Galois structures, in Lecture Notes on Noncommutative Geometry and Quantum Groups, Editor P.M. Hajac, notes by P. Witkowski, available at http://www.mimuw.edu.pl/ pwit/toknotes/toknotes.pdf.

[16] Brzeziński T., Majid S., Quantum group gauge theory on quantum spaces, Comm. Math. Phys. 157 (1993), 591-638, arXiv:hep-th/9208007.

[17] Brzeziński T., Majid S., Coalgebra bundles, Comm. Math. Phys. 191 (1998), 467-492, arXiv:q-alg/9602022.

[18] Brzeziński T., Majid S., Quantum geometry of algebra factorisations and coalgebra bundles, Comm. Math. Phys. 213 (2000), 491-521, arXiv:math.QA/9808067.

[19] Brzeziński T., Sitarz A., Dirac monopoles from the Matsumoto noncommutative spheres, in Group 24: Physical and Mathematical Aspects of Symmetries, Institute of Physics Conference Series, Vol. 173, Editors J.-P. Gazeau, R. Kerner, J.P. Antoine, S. Metens, J.Y. Thibon, IOP, Bristol and Philadelphia, 2003, 791-794, arXiv:math-ph/0304037.

[20] Cartan H., Seminaire Cartan, E.N.S. 1949/1950, W. A. Benjamin, Inc., New York - Amsterdam, 1967.

[21] Connes A., Dubois-Violette M., Noncommutative finite-dimensional manifolds. I. Spherical manifolds and related examples, Comm. Math. Phys. 230 (2002), 539-579, arXiv:math.QA/0107070. 
[22] Connes A., Landi G., Noncommutative manifolds, the instanton algebra and isospectral deformations, Comm. Math. Phys. 221 (2001), 141-159, arXiv:math.QA/0011194.

[23] Cuntz J., Quillen D., Algebra extensions and nonsingularity, J. Amer. Math. Soc. 8 (1995), 251-289.

[24] Da̧browski L., Grosse H., Hajac P.M., Strong connections and Chern-Connes pairing in the Hopf-Galois theory, Comm. Math. Phys. 220 (2001), 301-331, arXiv:math.QA/9912239.

[25] Eisenbud D., Commutative algebra with a view toward algebraic geometry, Graduate Texts in Mathematics, Vol. 150, Springer-Verlag, New York, 1995.

[26] Hajac P.M., Strong connections on quantum principal bundles, Comm. Math. Phys. 182 (1996), 579-617, arXiv:hep-th/9406129.

[27] Hajac P.M., A note on first order differential calculus on quantum principal bundles, Czechoslovak J. Phys. 47 (1997), 1139-1143, arXiv:q-alg/9708014.

[28] Hajac P.M., Krähmer U., Matthes R., Zieliński B., Piecewise principal comodule algebras, J. Noncommut. Geom. 5 (2011), 591-614, arXiv:0707.1344.

[29] Kassel C., Schneider H.-J., Homotopy theory of Hopf Galois extensions, Ann. Inst. Fourier (Grenoble) 55 (2005), 2521-2550, arXiv:math.QA/0402034.

[30] Landi G., An introduction to noncommutative spaces and their geometries, Lecture Notes in Phys. Monographs, Vol. 51, Springer-Verlag, Berlin, 1997, arXiv:hep-th/9701078.

[31] Landi G., van Suijlekom W., Principal fibrations from noncommutative spheres, Comm. Math. Phys. 260 (2005), 203-225, arXiv:math.QA/0410077.

[32] Majid S., Foundations of quantum group theory, Cambridge University Press, Cambridge, 1995.

[33] Schneider H.-J., Principal homogeneous spaces for arbitrary Hopf algebras, Israel J. Math. 72 (1990), 167195.

[34] Woronowicz S.L., Compact matrix pseudogroups, Comm. Math. Phys. 111 (1987), 613-665.

[35] Woronowicz S.L., Differential calculus on compact matrix pseudogroups (quantum groups), Comm. Math. Phys. 122 (1989), 125-170. 